\title{
Review, Design, Optimization and Stability Analysis of Fractional-Order PID Controller
}

\author{
Ammar SOUKKOU, M.C. BELHOUR \\ Faculty of Sciences and Technology, Department of Electronics, Jijel University, \\ P.O. Box 98, Ouled Aissa, Jijel 18000, Algeria. \\ E-mail: soukkou.amr@gmail.com; belhour.mohammed.cherif@gmail.com
}

Salah LEULMI

Department of Electric Power Engineering, Skikda Electric Power Systems Laboratory, University of August 20th, 1955, Skikda, Algeria.

E-mail: salah.leulmi@yahoo.fr

\begin{abstract}
This paper will establish the importance and significance of studying the fractional-order control of nonlinear dynamical systems. The foundation and the sources related to this research scope is going to be set. Then, the paper incorporates a brief overview on how this study is performed and present the organization of this study. The present work investigates the effectiveness of the physical-fractional and biological-genetic operators to develop an Optimal Form of Fractional-order PID Controller (O2Fo-PIDC). The newly developed Fo-PIDC with optimal structure and parameters can, also, improve the performances required in the modeling and control of modern manufacturing-industrial process (MIP). The synthesis methodology of the proposed O2Fo-PIDC can be viewed as a multi-level design approach. The hierarchical Multiobjective genetic algorithm (MGA), adopted in this work, can be visualized as a combination of structural and parametric genes of a controller orchestrated in a hierarchical fashion. Then, it is applied to select an optimal structure and knowledge base of the developed fractional controller to satisfy the various design specification contradictories (simplicity, accuracy, stability and robustness).
\end{abstract}

Index Terms-Optimal fractional-order controllers, BIBO stability analysis, Multiobjective genetic algorith m, CE150 Helicopter model.

\section{INTRODUCTION}

Systems and control theory has evolved as an important confluence between the engineering and mathematics disciplines. In general, it relates to the dynamical system analysis and behaviour and to the various techniques in which these systems can be influenced to obtain some desired results.

Actually, a wide variety of mathematical techniques are used in the field of control system design. Moreover, many control, modelling and optimization problems can be recast as a learning problem and be solved with appropriate mathematical tools. It has been shown that famous ordinary-order PID controllers (Oo-PIDC), generally, do not work well for nonlinear complex and vague systems that have no precise mathematical models.

However, a class of newly modified-type of Oo-PID controller based on advanced and intelligent approaches has been designed and simulated for this purpose.

Recently, fractional-order calculus, included in advanced techniques, has received an increasing interest due to the fact that fractional operators are defined by natural phenomena [1].

Fractional derivatives and integrals operators ${ }_{c} D_{t}^{ \pm \alpha} f(t), \alpha \in \mathbb{R}^{+}$provide an excellent instrument for the description of memory and hereditary properties of various materials and processes.

The theoretical and practical interest of these operators is nowadays, well established. Its applicability to science and engineering can be considered as an emerging new topic. It includes some areas, such as biology and chemical engineering (irrigation canal control [2], low pressure flowing water network [3], networked control system [4]), control and simulation of photovoltaic-wind energy systems [5, 6], hydraulic turbine regulation [7], flight control [8], fractional-order chaotic systems modeling and control [9, 10], Modeling of Economic Order Quantity [11], control of a servo systems [12, 13], realization of higher-order filters [14], robotic control [15], astronomy [16], .. etc.

The fractional-order Controllers ( $\mathrm{FoC}$ ) were introduced firstly by Prof. Oustaloup [17]. He developed the socalled CRONE controller, French abbreviation of "commande Robuste d'ordre non entier". The idea of using $\mathrm{FoC}$ for the dynamic system control is well addressed in $[18,19]$. More recently, Podlubny proposed a generalization of the Oo-PIDC, as a natural extension to the Fo-PIDC or a $\mathrm{PI}^{\lambda} \mathrm{D}^{\mu}$ controller, involving an integrator of $\lambda \in \mathbb{R}^{+}$order and a differentiator of $\mu \in \mathbb{R}^{+}$ order. There are many variants of FoC that have been used in different applications depending on the application specific require ments: CRONE controller and its three generations [18], Fo-PIDC [20], non-integer integral [21] and tilted proportional and integral 
compensator [22]. Other FoC like fractional-order leadlag compensator [23] and fractional-order phase shaper are also becoming popular in recent robust process control applications [24]. The most common is the family of the Fo-PID controllers.

The application of fractional-order calculus has been significantly increased and the FoC are, attractively, becoming a major topic of control. However, designing high-performance and cost effective controllers is a very complex task which could be, practically, impossible to optimally accomplish by some conventional trial and error methods considering different design criterion in time and frequency-domain.

There are many methods to construct and design such FoC which can be regarded as analytical and learning problems of a desired performance of the controlled systems

- The first step is to define a mathematical structure of the controller based on the application of approximation (discretization) methods of the fractional operators $c^{ \pm} D_{t}^{ \pm \alpha} \cdot \alpha \in \mathbb{R}^{+}$(resp. $s^{ \pm \alpha}$ ) based on the analysis field (time or frequencydomain).

- The second step is to identify the gains 'parameters' of the predefined structure. After an extensive literature search on methods of tuning and optimization of FoC, especially the Fo-PIDC, we arrived to classify these methods, according to the

\section{$\checkmark \quad$ Manual methods (trial-and-error) [25];}

$\checkmark$ Analytical methods (phase and gain marginbased specifications [3], flat phase [26], dominant roots-pole placement [27, 28], internal model control (IMC) [29]);

$\checkmark$ Optimization-based methods (F-MIGO Algorithm [23], Monje's methods [3], simplex search minimization [3], metaheuristic algorithms (GA [4, 30], PSO [31], DE [4], SA [32], ABCA [12], IWO [33], FOA [34], CSA [15]) and least squares optimization method [35]);

$\checkmark \quad$ Tuning rule for plants (with an S-shaped step response - with a critical gain) [29, 36];

$\checkmark \quad$ Auto tuning [3, 21].

Based on the previous classification, we may notice that metaheuristic algorithms 'evolutionary algorithms (EA)', especially GA, PSO and DE, have the majority of references in the design of the predefined (characterized) FoC. The frequency-domain analys is is the dominant case of these applications, where the problem is how to approximate the integrator-derivator operator $s^{ \pm \alpha}$ and how to optimize the associated parameters of the controller under development?

This research is aimed for appreciation of the fractional calculus, coupling fractional calculus-intelligent design and optimization approaches Thus, it is made as an application in engineering field. The present work investigates the effectiveness of the physical and biological-based operators (fractional and genetic approaches) to develop an optimal form (structure with optimal dimension)) of fractional-order proportionalintegral-derivative controller (O2Fo-PIDC) with optimal parameters (knowledge base) and able to improve the performances required in the modelling and control of industrial process. In this context, issues related to the design of the proposed controller can be classified into mathematical configuration and parametric optimization categories.

- Characterization 'mathematical configuration' of the control law under development by a judicious choice of discretization techniques of derivative

$$
\begin{aligned}
& \beta \cdot{ }_{c} D_{t}^{\alpha} f(t),(\beta, \alpha) \in \mathbb{R}^{+} \quad \text { and integral } \\
& \gamma \cdot{ }_{c} I_{t}^{\lambda} f(t)=\gamma \cdot{ }_{c} D_{t}^{-\lambda} f(t),(\gamma, \lambda) \in \mathbb{R}^{+} \text {operato }
\end{aligned}
$$
rs involved in modeling of the control signals. In addition, it is indispensable to take into account the type of the association (parallel or serial) of various proportionality, derivative and integral actions. It depends on the design field, timedomain (control of nonlinear dynamical continuous or discrete systems) or frequencydomain (control of linear continuous systems). In this phase, the constraint of computing time (long memory effect) related to an analytical model of fractional systems will be treated with prudence and is also the seeking tools to improve the tradeoff between simplicity and accuracy specifications.

- The learning process involves the development or the implementation of the learning and optimization algorithms of relevant parameters of fractional-order control system under develop ment. This transaction is based upon the factors of accuracy and stability conditions of the control loop and the used optimization algorithms (conventional, advanced or intelligent-based approaches).

The paper is organized as follows. Section 2 provides fundamentals of fractional calculus and its properties in time and frequency-domain. A presentation of the FoPIDC, their description and properties are also included.

A survey of various design and optimization approaches of Fo-PIDC is considered. The aim of Section 3 is to design an optimal and simplified form of Fo-PIDC by considering various contradictory objective functions to control dynamical systems, such as simplicity, efficiency (precision) and stability. The design deal with time-domain analysis of the O2Fo-PIDC adapted to nonlinear dynamical systems. In section 4, some simulation results to illustrate the effectiveness and robustness of the proposed control system are displayed. Finally, we present some conclusions and the contributions developed in this research. 


\section{Fractional CAlCulus: An OVERVIEW}

Fractional calculus, capable of representing natural phenomena in a more general way and do not approximate the processes by considering that the order of the governing differentials are integers only [23, 37], is a branch of mathematical analysis. It studies the possibility of tacking real numbers power of the differential and integration operator. The generalized differ-integrator may be put forward as [38]

$$
\begin{aligned}
{ }_{c} D_{t}^{\alpha} f(t) & ={ }_{c} I_{t}^{-\alpha} f(t)=D_{t-c}^{\alpha} f(t) \\
& =\frac{d^{\alpha} f(t)}{[d(t-c)]^{\alpha}}= \begin{cases}d^{\alpha} f(t) / d t^{\alpha}, & \alpha>0 \\
1, & \alpha=0 \\
\int_{c}^{t} f(t) d \tau^{-\alpha} & , \alpha<0\end{cases}
\end{aligned}
$$

where $\alpha \in \mathbb{R}^{+}$represents the real-order of the differintegral, $t$ is the parameter for which the differ-integral is taken and $c$ is the lower limit, generally $c \geq 0$ for the causal systems. Several alternative definitions of the fractional-order integrals and derivatives exist. The three most common known definitions for fractional derivatives-integrals are Grünwald-Letnikov (GL) definition, Riemann-Liouville (RL) definition and Caputo (C) definition, given, in time-domain, as follows

- Grünwald-Letnikov definition

$$
G L_{c} D_{t}^{\alpha} f(t)=\lim _{h \rightarrow 0} h^{-\alpha} \cdot \sum_{j=0}^{N-1}(-1)^{j} \cdot\left(\begin{array}{c}
\alpha \\
j
\end{array}\right) \cdot f(t-j \cdot h)
$$

where $N=\left[\frac{t-c}{h}\right]$ is the upper limit of the computational universe, where $[\bullet]$ means the integer part, $h$ is a grid size and $\left(\begin{array}{c}\alpha \\ j\end{array}\right)$ is a binomial coefficients.

- Riemann-Liouville definition given by the generalized form

$$
\begin{aligned}
{ }_{c} L_{t} D_{t}^{\alpha} f(t)= & \frac{1}{\Gamma(n-\alpha)}\left(\frac{d}{d t}\right)^{n} \int_{c}^{t} \frac{f(\tau)}{(t-\tau)^{1-(n-\alpha)}} d \tau, \\
& (n-1)<\alpha<n
\end{aligned}
$$

where $\Gamma(\bullet)$ is the Euler Gamma function.

- Caputo definition, which so metimes called smooth fractional derivative, defined by

$$
\begin{aligned}
{ }_{c}^{C} D_{t}^{\alpha} f(t)= & \frac{1}{\Gamma(n-\alpha)} \int_{c}^{t} \frac{f^{(n)}(\tau)}{(t-\tau)^{1-(n-\alpha)}} d \tau, \\
& (n-1)<\alpha<n
\end{aligned}
$$

As shown by the RL and GL definitions, the fractionalorder derivatives are global operators having a memory of all past events. This property is used to model hereditary and memory effects in most materials and systems [13]. For numerical calculation of fractionalorder derivative, we can use the relation (2) derived from the GL definition. This approach is based on the fact that for a wide class of functions, GL (2), RL (3) and Caputo's (4) are equivalent [10]. The fractional-order derivative and integral can also be defined in the transformation-domain. It is shown that the Laplace transform of a fractional derivative of a signal $f(t)$ is given by

$$
\begin{aligned}
L\left\{D_{t}^{ \pm \alpha} f(t)\right\}= & s^{ \pm \alpha} \cdot \underbrace{L\{f(t)\}}_{F(s)}- \\
& \sum_{k=1}^{n-1} s^{k} \cdot \underbrace{\left(\left.0 D_{t}^{ \pm \alpha-k-1} f(t)\right|_{t=0}\right)}_{\text {Initialization function }}
\end{aligned}
$$

Considering null in itial conditions, the last expression (5) is reduced to the following suitable form:

$$
L\left\{D_{t}^{ \pm \alpha} f(t)\right\}=s^{ \pm \alpha} \cdot F(s)
$$

The Laplace transform reveals to be a valuable tool for the analysis and design of fractional-order control systems for reasons of analysis and synthesis simplicity [13]. It is noted that some MATLAB-tools of the fractional-order dynamic system modeling, control and filtering can be found in [39, 40]. Numerical methods for simulating and discretization fractional-order systems are given in detail in [10] and [41].

A. Approximation and numerical implementation 'discretization' of fractional operators: An overview

The ideal digital fractional-order differentiatorintegrator $0 D_{t}^{ \pm \alpha}$ is a discrete-time whose output $y(n)$ is uniformly sampled version of the $\alpha^{\text {th }}$-order derivative of $f(t)$. Henceforth, we assume that $\alpha>0$, we write

$$
y(n)=\left.{ }_{0} D_{t}^{ \pm \alpha} f(t)\right|_{t=n h}
$$

In general, if a function $f(t)$ is approximated by a grid function, $f(n \cdot h)$, where $h$ is the period of 
discretization (step size of calculation or sampling time). The approximation for its fractional derivative order $\alpha$ can be expressed as [42]

$$
\begin{aligned}
y_{h}(n h)=\frac{d^{ \pm \alpha} f(t)}{d t^{ \pm \alpha}} & =\lim _{h \rightarrow 0} h^{\mp \alpha} \cdot[\Delta f(t)]^{ \pm \alpha} \\
& =h^{\mp \alpha} \cdot\left(w\left(\zeta^{-1}\right)\right)^{ \pm \alpha} \cdot f_{h}(n \cdot h)
\end{aligned}
$$

where $\left.\Delta f(n \cdot h)\right|_{t=n \cdot h}=f(n \cdot h)-f(n \cdot h-h), \zeta^{-1}$ is the shift operator and $w\left(\zeta^{-1}\right)$ is a so-called generating function (GF). This GF and its expansion determine both the form of the approximation and the coefficients [43].

Fractional derivative and integral operators are often difficult to find analytically and the simulation of fractional systems is complicated due to their long memory behaviour as shown by Oustaloup [18]. A number of techniques are available for approximating fractional derivatives and integrals. These latter can be classified into three main groups based on the field under study, temporal (t-plane), frequency (s-plane) and timediscrete (z-plane) analysis as illustrated in figure 1.

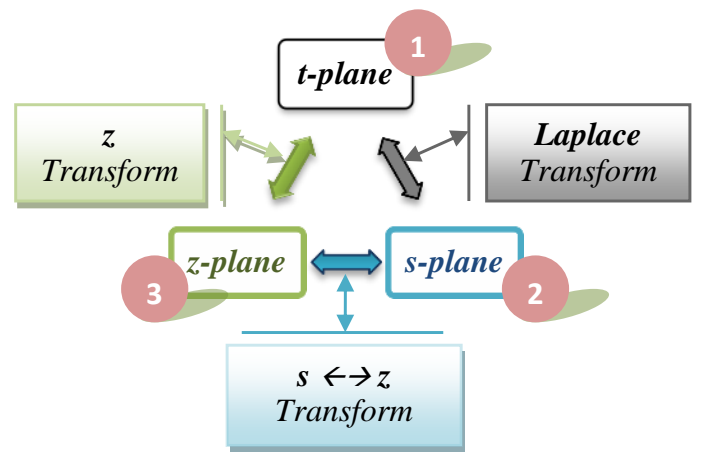

Fig.1. Simulation-discretization tools of fractional operators.

1. Methods based on the computation of analytical expression of derivative and integral signal (temporal-analysis). The GL (2) and the AdamsBashforth-Moulton (ABM) algorithms based on the Caputo's definition are the most used methods.

2. Methods based on the approximation of a fractional model by a rational continuous-time one (frequency-analysis). The Laplace transform is the most used algorithm. The main steps to compute the general formula of the output are:

Step 1: Compute the Laplace transform from Eq. (5).

Step 2: Evaluate $s^{ \pm \alpha}=\phi(s)=N(s) / D(s)$ by using the most used $s^{ \pm \alpha}$ approximation and can be classified into the three following categories [44]:

a. Methods based on mathematical techniques for rational approximation of functions [45, 46]. These techniques are based on the approximation of an irrational function, $G(s)$, by a rational one defined by the quotient of two polynomials in the variable $s$.

$b$. Methods based on the explicit recursive location of poles and zeros of the rational approximation. Many iterative techniques exist for realization of the fractional elements in continuous time. The most used are Oustaloup [18], Charef [47], Carlson [48] and Matsuda [49].

c. Methods based on frequency domain identification, that is, on finding a rational integer-order system whose frequency response fits that of the fractional-order operator [45]. Generally, linear least squares and metaheuristic optimization techniques are proposed to solve the frequencydomain identification problem $[50,51]$.

Step 3: Replace the last approximation of $s^{ \pm \alpha}$ and evaluate the inverse of Laplace operator $L^{-1}\left\{L\left\{{ }_{0} D_{t}^{ \pm \alpha} f(t)\right\}\right\} \quad$ in (5) to obtain the expression to simulate the fractional derivator and integrator of signal $f(t)$.

3. Methods based on the approximation of a fractional model by a rational discrete-time one (discrete-time analysis). The z-transform is the most used algorithm. The main steps to compute the general formula of the output are:

Step 1: Discretization of the operator $s$ by $s=\psi\left(z^{-1}\right)$ and then the approximation of a GF of the form $\left(\psi\left(z^{-1}\right)\right)^{ \pm \alpha}$ corresponding to $s^{ \pm \alpha}$. The most used $s$-to- $z$ transform $s^{ \pm \alpha}$ are [52]: Euler (backward - forward), Tustin, Al-A laoui, Simpson, Al-Alaoui - Simpson, Al-Alaoui - SKG and AlAlaoui - Schneider.

Step 2: To obtain the coefficients of the approximation equations for fractional calculus $\left(\psi\left(z^{-1}\right)\right)^{ \pm \alpha}$, we can perform the most used algorithms:

$\checkmark \quad$ The direct power series expansion (PSE) of the Euler operator [53, 54] (FIR filter structure);

$\checkmark$ The continuous fractional expansion (CFE) of the Tustin operator [53, 54] (IIR filter structure); or

$\checkmark$ The numerical integration-based method [55, 56].

Step 3: Evaluate the inverse operator $Z^{-1}\left\{Z\left\{{ }_{0} D_{t}^{ \pm \alpha} f(t)\right\}\right\}$ to obtain the approximation of ${ }_{0} D_{t}^{ \pm \alpha} f(t)$. 
From a control and signal processing perspective, the GL and ABM approaches seem to be the most useful and intuitive, particularly, for a discrete-time implementation. Moreover, in the analysis and design of control systems, we usually adopt the Laplace transform method. As a result, fractional-order controller synthes is is preferably carried out in the frequency domain. This is the reason why the most design methods proposed, so far, for FoC are based on using frequency-response information.

\section{B. Fractional-order PID controller}

The motivation on using the digital Fo-PIDC was that Oo-PIDC belongs to the dominating industrial controllers. Therefore, there is a continuous effort to improve their quality and robustness. The traditional Oo-PIDC which involves proportional (with gain $K_{P}$ ) plus integral (with gain $K_{I}=K_{P} \cdot T / T_{i}$ ) plus derivative (with gain
$K_{D}=K_{P} \cdot T_{d} / T$ ) actions based on the error signal, given in continuous and discrete-time as

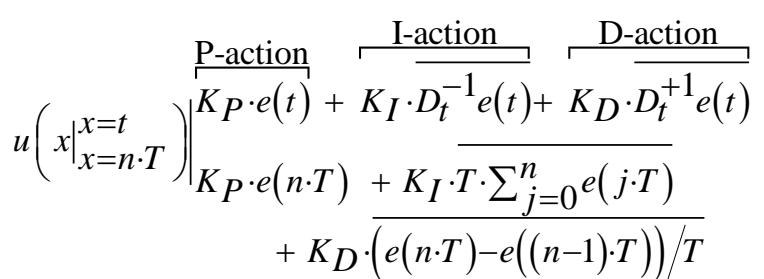

can be generalized to a fractional controller involving an integrator of order $\lambda \in \mathbb{R}^{+}$and a differentiator of order $\mu \in \mathbb{R}^{+}[19,20]$. Figure 2 indicates the schematic diagram of the Fo-PIDC loop.

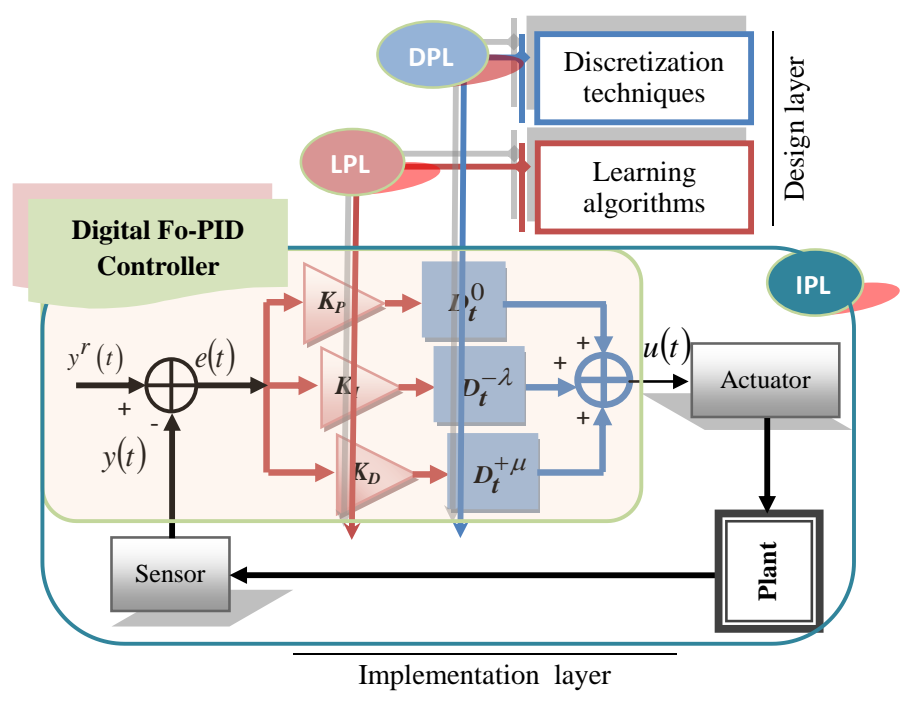

Fig.2. Fo-PIDC loop design.

The mathematical model of the Fo-PIDC described in continuous and discrete-time is given by

$$
u=\mid \begin{aligned}
& K_{P} \cdot e(t)+K_{I} \cdot D_{t}^{-\lambda} e(t)+K_{D} \cdot D_{t}^{\mu} e(t) \\
& K_{P} \cdot e(n \cdot T)+K_{I} \cdot \Delta_{n \cdot T}^{-\lambda} e(n \cdot T)+K_{D} \cdot \Delta_{n \cdot T}^{\mu} e(n \cdot T)
\end{aligned}
$$

To analyze and simulate complex dynamical systems, variable-order fractional is proposed by $\mathrm{Hu}$ Sheng et al. [57] as a generalization of the constant-order $\mathrm{PI}^{\lambda} \mathrm{D}^{\mu}$ controller, where $\{\lambda, \mu\}$ in (10) are replaced by $\{\lambda(t), \mu(t)\}$.

As shown in Fig. 2, it consists of two layers: a computational layer (design) and the decision layer (operational).

Three main stages are necessary for the implementation of the control loop:
- The first is to define an analytical structure of the controller by applying the methods of discretization and approximation of fractionalorder operators.

- The second consists of determining the knowledge base of the controller structure, previously identified, by application of learning and optimization algorithms.

- The last phase is the verification of the coherence of the proposed algorithm by real-time execution.

The frequency-domain analysis is the most used in the design of the FoC, where the continuous transfer function of the Fo-PIDC is obtained through Laplace transform or discrete $z$-transfer function.

- Laplace transform of transfer function of system with input $E(s)$ (error signal) and controller's output $U(s)$, as 


$$
\begin{gathered}
G_{C}(s)=\frac{U(s)}{E(s)}=K_{P}+K_{I} \cdot s^{-\lambda}+K_{D} \cdot s^{\mu} \\
\{\lambda, \mu\}>0
\end{gathered}
$$

- Discrete transfer function given by the following expression:

$$
\begin{gathered}
G_{c}(z)=K_{P}+K_{I} \cdot\left(\psi\left(z^{-1}\right)\right)^{-\lambda}+K_{D} \cdot\left(\psi\left(z^{-1}\right)\right)^{\mu} \\
\{\lambda, \mu\}>0
\end{gathered}
$$

where $s \rightarrow \psi\left(z^{-1}\right)$ denotes the discrete operator, expressed as function of the complex variable $z$ or the shift operator $z^{-1}$. The fractional differentiatorintegrator $s^{ \pm \alpha}$ is substituted by its discrete-time equivalent $\left(\psi\left(z^{-1}\right)\right)^{ \pm \alpha}$.

From figure 3(a), it can be remarked that the Oo-PIDC can only be switched in four conditions: $\{P, P I, P D, P I D\}$. It restricts the controller performance. Relatively, depending on the value of orders $\{\lambda, \mu\}$ ( shaded area represented in figure $3(\mathrm{~b})$ ), we get an infinite number of choices for the controller's type, defined continuously on the $(\lambda, \mu)$-plane, i.e., The Fo-PIDC expands the OoPIDC from point to plane, thereby it adds the flexibility to controller design and allows us to control our real world processes more accurately [38, 58]. In [59], the authors applied the $\mathrm{P}^{\alpha} \mathrm{I}^{\lambda} \mathrm{D}^{\mu}$ controller to enhance the performance of the force feedback control system, where the pair $\left\{K_{i}, i=\alpha, \lambda, \mu\right\}$ represents the control gain and the non-integer order. Thus, the design procedure involves the parameters of a FoC with three terms $\left\{K_{\alpha} \cdot e^{\alpha}(t), K_{\lambda} \cdot D^{\lambda} e(t), K_{\mu} \cdot D^{-\mu_{e}(t)}\right\}$ as sociated with the $\mathrm{P}, \mathrm{I}$ and $\mathrm{D}$-actions, respectively. This last form expands the Oo-PIDC from point to $(\alpha, \lambda, \mu)$-plane as shown in figure 3 (c).

Fo-PID controllers have received a considerable attention in the last years both from academic and industrial point of view. However, due to the complexity of the fractional-order systems, these control design techniques available for the ubiquitous fractional-order systems suffer from a lack of direct systematic approaches based on the fair comparison with the traditional integer-order controllers [58], [60, 61]. Searches in the field of fractional control systems are concentrated around two areas

- Development of tools for calculating (discretizing) fractional operators $D_{t}^{ \pm \alpha} \cdot, \alpha \in \mathbb{R}$ (resp. $s^{ \pm \alpha}$ ) based on the used analysis field under study (time or frequency-domain).

- Development or implementation of the learning approaches of relevant parameters of control system under development (tuning and optimization).

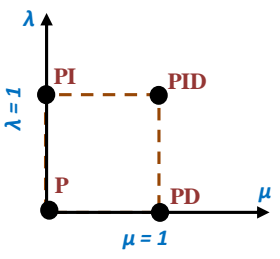

(a)

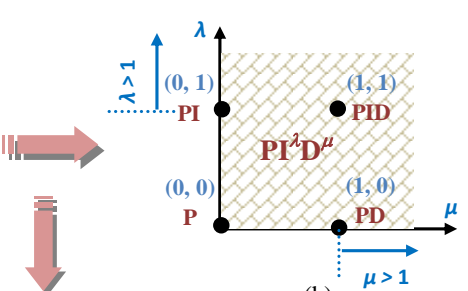

(b)

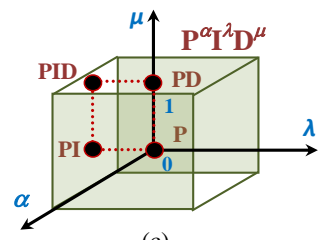

(c)

Fig.3. Generalization of the Fo-PIDC

\section{CONTROLLER DESIGN}

This section should address the most significant is sues schematized in figure 2 by codes into circles DPL, LPL and IPL, respectively.

- The main question in DPL is pronounced as: Is it possible to analytically design the Fo-PIDC with the best achievable performances, such as simplicity and accuracy?

- The aim of LPL: It is how this Fo-PIDC can be tuned for quantitative performances, such as accuracy, stability and robustness?

- The problem of IPL is pronounced as: What a performance limit does the Fo-PIDC have?

\section{A. Problem formulation and adopted tools}

The fractional calculus has received an increasing interest due to the fact that fractional operators are defined by natural phenomena [1]. In the implementation of the fractional controllers, especially Fo-PIDC finite order approximation is required since fractional elements have infinite order. This difficulty is caused by the mathematical nature of the fractional order operators which are defined by convolution and require 'unlimited memory'. During the synthesis of the proposed control law, the required performances are articulated around various contradictory objective functions, such as:

$\checkmark$ Simple and optimal analytical structure and parameters;

$\checkmark$ Efficiency and precision;

$\checkmark \quad$ Stability and robustness against disturbances;

$\checkmark$ Implementation ability in real-time. 
In this context, issues related to the design of the proposed controller can be classified into mathematical modeling 'characterization' of the control law under development 'analytical structure' and identification 'learning' of the knowledge base associated to the predefined analytical model of controller 'Parametric optimization'.

\section{B. Mathematical background: An overview}

The Oo-PIDC algorithm is one of the most commonly used control algorithms in industry. It's considered as a generic closed loop feedback mechanism. The Oo-PIDC output is computed in continuous time as illustrated in (9). For a small sample increment $T>0$, (9) can be turned into a difference equation by discretization [62]. It can be predicted that (9) needs a large quantity of memory space 'RAM' to store the results of the computation, i.e, to compute the sum $\sum_{j=0^{\bullet}}^{n}$, all past errors $\{e(0), e(1), \cdots, e(n)\}$, have to be stored.

This algorithm is called the 'position algorithm' [62]. To avoid the redundancy and to derive the recursive algorith $\mathrm{m}$, we can apply the integer derivative operator in (9), as given by

$$
\begin{aligned}
d u(t) / d t= & K_{P} \cdot d e(t) / d t+K_{I} \cdot e(t) \\
& +K_{D} \cdot d / d t(d e(t) / d t)
\end{aligned}
$$

In order to take better advantage of discrete form of the data, (13) has been approximated by the following difference equations based on the first-order Euler approximation approach

$$
\begin{gathered}
\qquad x(t) / d t \cong \lim _{T \rightarrow 0}(x(t)-x(t-T)) / T \\
d^{2} x(t) / d t^{2}=d / d t(d x(t) / d t) \\
\cong \lim _{T \rightarrow 0}((x(t)-x(t-T)) / T-(x(t-T)-x(t-2 \cdot T)) / T) / T
\end{gathered}
$$

Replace (14) and (15) in (13), we obtain the approximate expression of the Oo-PIDC in time-domain as

$$
\begin{array}{r}
u(t) \cong u(t-T)+K_{0}(\cdot) \cdot e(t)+K_{1}(\cdot) \cdot e(t-T) \\
+K_{2}(\cdot) \cdot e(t-2 \cdot T)
\end{array}
$$

and,

$$
\left\{\begin{array}{l}
K_{0}(\cdot)=\left\{K_{P}+K_{I} \cdot T+K_{D} \cdot T^{-1}\right\} \\
K_{1}(\cdot)=\left\{-K_{P}-2 \cdot K_{D} \cdot T^{-1}\right\} \\
K_{2}(\cdot)=\left\{K_{D} \cdot T^{-1}\right\}
\end{array}\right.
$$

Use a series of discrete sampling point $t \approx n \cdot T, \quad(n=0,1,2, \cdots) \quad$ to instead the recursive algorith $\mathrm{m}$, the steady-state value of Oo-PIDC process is obtained as

$$
\begin{aligned}
u(n \cdot T) & =u((n-1) \cdot T)+K_{0}(\cdot) \cdot e(n \cdot T) \\
& +K_{1}(\cdot) \cdot e((n-1) \cdot T)+K_{2}(\cdot) \cdot e((n-2) \cdot T)
\end{aligned}
$$

As shown in (17), the calculus of the $u(n \cdot T)$ is, mathematically, based on the $u((n-1) \cdot T), e(n \cdot T)$, $e((n-1) \cdot T)$ and $e((n-2) \cdot T)$. In the software imple mentation, the incremental algorith $\mathrm{m}$ (17) can avoid accumulation of all past errors $e(n)$ and can realize smooth switching from manual to automatic operation, compared with the position algorithm [62]. It is observed that in (17), the efficiency and the flexibility of the operating environment can be improved than (9) [63]. Many tuning methods are presented in literatures during this last decade that are based on a few structures of the process dynamics.

As mentioned above, the conventional Oo-PIDC (9) can be generalized to a FoC involving an integrator of order $\lambda \in \mathbb{R}^{+}$and a differentiator of order $\mu \in \mathbb{R}^{+}$as given in (10). Applied the derivative of the expression (10), we get

$$
D_{t}^{1} u(t)=K_{P} \cdot D_{t}^{1} e(t)+K_{I} \cdot D_{t}^{1-\lambda} e(t)+K_{D} \cdot D_{t}^{1+\mu_{e}(t)}
$$

In discrete-time control systems, it is required that the data are in form of the sampled data. Thus, it needs to discretize the fractional derivative or integral operation to realize the discrete-time fractional-order control. In general, the GL defin ition given in (2) is the most suitable method for the realization of discrete control algorith ms. In $(2),(-1)^{j} \cdot\left(\begin{array}{c}\alpha \\ j\end{array}\right)$ represents the binomial coefficients $c_{j}^{(\alpha)},(j=0,1,2, \cdots)$, calculated according to the relation

$$
c_{j}^{(\alpha)}= \begin{cases}0, & j<0 \\ 1, & j=0 \\ \left(1-\frac{1+(\alpha)}{j}\right) \cdot c_{j-1}^{(\alpha)}, & \forall j>0\end{cases}
$$

Replacing the analytical expressions of $D_{t}^{1-\lambda} x(t)$, $D_{t}^{1+\mu} x(t)$ computed from (2) and $D_{t}^{1} x(t)$ from (14) in 
(18), we can obtain the approximate expression of the FoPIDC in time-domain as

$$
\begin{aligned}
& u(t) \cong u(t-T)+K_{P} \cdot(e(t)-e(t-T)) \\
& \quad+\sum_{j=0}^{[t / T]}\left(K_{I} \cdot T^{\lambda} \cdot d_{j}+K_{D} \cdot T^{-\mu} \cdot q_{j}\right) \cdot e(t-j \cdot T)
\end{aligned}
$$

The coefficient $d_{j}$ and $q_{j}$ are computed from (19), respectively, and given by:

$$
\begin{cases}d_{j}= \begin{cases}1, & j=0, \\ \left(1-\frac{(2-\lambda)}{j}\right) \cdot d_{j-1}, & j=1,2, \ldots\end{cases} \\ q_{j}= \begin{cases}1, & j=0, \\ \left(1-\frac{(2+\mu)}{j}\right) \cdot q_{j-1}, & j=1,2, \ldots\end{cases} \end{cases}
$$

Using series of discrete sampling point $t \approx n \cdot T, \quad(n=0,1,2 ; \cdot \cdot) \quad$ instead of the recursive algorith $m$, the final expression of Fo-PIDC obtained after simplification of (20) is given by the generalized form

$$
\begin{aligned}
& u(n \cdot T)=u((n-1) \cdot T)+ \\
& \underbrace{\tilde{K}_{0}(\cdot) \cdot e(n \cdot T)+\tilde{K}_{1}(\cdot) \cdot e((n-1) \cdot T)+\tilde{K}_{2}(\cdot) \cdot e((n-2) \cdot T)}_{\Delta \tilde{u}_{\text {base }}(n \cdot T)} \\
& +\underbrace{\tilde{K}_{3}(\cdot) \cdot e((n-3) \cdot T)+\cdots+\tilde{K}_{n}(\cdot) \cdot e(0)}_{R_{L}^{\infty}(e(\cdot))}
\end{aligned}
$$

and,

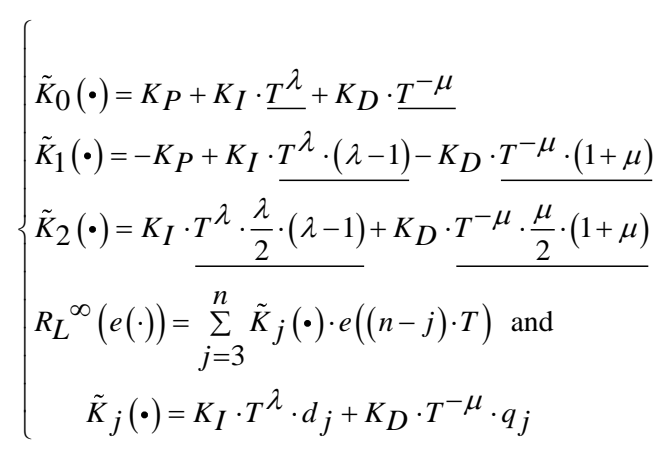

This expression is similar (in form) to that given by the relation (17) with the addition of a correction term $R_{L}{ }^{\infty}(e(\cdot))$ which represents an infinite sequence of linear regulators.

In the case of controller implementation, it is necessary to take into account some important considerations [45].

First of all, the value of $T$, the step when dealing with numerical evaluation is limited by the characteristics of the microprocessor-based system, used for the controller implementation, in two ways
- Each microprocessor-based system has its own minimum value of the sample period;

- Then, it is necessary to perform all the computations required by the control law between two samples.

Due to this last reason, it is very important to obtain good approximations with a minimal set of parameters. On the other hand, when the number of parameters in the approximation increases, it increases the amount of the required memory too. From Eq. (22), concluding remarks can be pronounced.

- The sampling time $T$ determines the rate at which the feedback gains are updated. Faster sampling rate $(T<<)$ allow higher adaptation rate to be used, which in turn leads to a better tracking performance. Therefore, it is difficult to implement the algorithm in real-time because of the existence of the term $R_{L}^{\infty}(e(\cdot))$ in (22). Cumulative values represent a constraint for the implementation of the algorith $m$ in real-time. The problem of running time in the phase of design and simulation is, also, a problem to report (alert).

- In general, when the sampling rate is slow (large $T)$, the effects of sampling and discretization are more pronounced and the control system performance is degraded. Therefore, in practical implementation, it is highly desirable to increase the sampling rate as much as possible by optimizing the real-time control program or using a multiprocess or parallel computing system.

\section{Elaboration of the proposed Fo-PID model}

In practical applications of the Fo-PIDC (22), real-time realization problems arise due to linearly growing processed samples (known as the growing calculation tail') and finite microprocessor memory. The prag matic solution is to adapt the digital control algorithm to the dynamic characteristics of the process under study. In order to

$\checkmark \quad$ Reduce the amount of historical data;

$\checkmark$ Improve the accuracy of the numerical solution;

$\checkmark$ Avoid the redundancy;

$\checkmark$ Then, derive the recursive algorithm in its optimal form,

a simplified analytical form 'structure' of the Fo-PIDC (22) is proposed. The main idea is developed by SOUKKOU et al. in [64]. It consists to make the correction term $R_{L}{ }^{\infty}(e(\cdot))$ in (22) adjustable (manually or automatically) accord ing to the physical characteristics of the system to be controlled and technological performances of the monitoring PLC (storage space speed) used to implement the control algorithm. Eq. (22) can, also, be rewritten in the following manner 


$$
\begin{aligned}
& u(n \cdot T) \cong u((n-1) \cdot T) \\
& +\underbrace{\tilde{K}_{0}(\cdot) \cdot e(n \cdot T)+\tilde{K}_{1}(\cdot) \cdot e((n-1) \cdot T)+\tilde{K}_{2}(\cdot) \cdot e((n-2) \cdot T)}_{\Delta \tilde{u}_{i, j}(n \cdot T),(i=0 ; j=\{0,1,2\})} \\
& +\underbrace{\tilde{K}_{3}(\cdot) \cdot e((n-3) \cdot T)+\tilde{K}_{4}(\cdot) \cdot e((n-4) \cdot T)+\tilde{K}_{5}(\cdot) \cdot e((n-5) \cdot T)}_{\Delta \tilde{u}_{i, j}(n \cdot T),(i=1 ; j=\{3,4,5\})} \\
& +\underbrace{\tilde{K}_{6}(\cdot) \cdot e((n-6) \cdot T)+\tilde{K}_{7}(\cdot) \cdot e((n-7) \cdot T)+\tilde{K}_{8}(\cdot) \cdot e((n-8) \cdot T)}_{\Delta \tilde{u}_{i, j}(n \cdot T),(i=2 ; j=\{6,7,8\})}
\end{aligned}
$$

Compared with (17), the expression (23) can be seen as a combination of a large set of linear PID regulators, temporarily shifted with moderate gains $\tilde{K}_{i}(\bullet),(i=0,1, \cdots, n)$. In (23), each $i^{\text {th }}$ iteration requires the re-calculation and summation of every previous time point convolved with $\tilde{K}_{i}(\bullet)$. This becomes increasingly cumbersome for large times, which requires significant nu mbers of computations and memory storage requirements.

Applying some ideas as, for instance, short memory principle [19], we can reduce the computational cost of time-domain methods. Short memory principle means taking into account the function behavior only in the "recent past". The results obtained by these methods are more reliable than those determined using the frequency based approximation [10]. This would then result in approximating (22) by truncating backwards summation, only taking into account times on the interval $[t, L \cdot T]$ instead of $[0, t]$, where $L$ is defined as the memory length.

According to the short memory principle [19], the length of the system memory can be substantially reduced in the numerical algorithm to get reliable results. Thus, $N$ in (2) becomes $N \rightarrow N(t)=\min ((t-L) / h, L / h)$. Obviously, for this simplification, we pay a penalty in the form of some inaccuracy. If $|f(t)| \leq M$, we can easily establish the following estimate for determining the memory length $L$, providing the required accuracy $\varepsilon$ $L \geq(M /(\varepsilon \cdot|\Gamma(1-\alpha)|))^{1 / \alpha}$ and the upper limit of summation $N$ is $N \geq\left(M /\left(T^{\alpha} \cdot|\varepsilon \cdot \Gamma(1+q)|\right)\right)^{1 / \alpha}$. The upper limit of summation $N$ in (2) can be calculated from the inhomogeneous sampling algorithm proposed by Fujio Ikeda in [65] as $N \leq\left[L^{\alpha} /(\Delta T \cdot \Gamma(1+\alpha))\right]$, where $[x]$ means truncation to $x(\in \mathbb{R}), L$ is the calculation window limiting to some fixed time spam and $\Delta T$ is the 'transformed time' sampling period. $\Gamma(\bullet)$ is the Euler Gamma function given by $\Gamma(1+\alpha \cdot k) \cong(\alpha \cdot k) !, \forall k \in \mathbb{N}$.

Brian P. Sprouse et al. [66] introduce an adaptive timestep method for computing the contribution of the memory effect associated with the history of a system, where fractional methods must be taken into account. Zhe Gao et al. [67] proposed an alternative discretization algorith $m$ based on the Haar wavelet method and reduces the store space of the history data, just calculating the fractional order integral with five sampled data $f(n \cdot T), f((n-1) \cdot T), \quad f((n-2) \cdot T), \quad f((n-3) \cdot T)$ $f((n-4) \cdot T)$ and the initial value $f(0)$.

The present work represents a newly powerful and simple approach to provide a reasonable tradeoff between computational overhead, storage space and numerical accuracy. Rearranging (23), the discrete Fo-PIDC law can be rewritten as:

$$
\begin{aligned}
& u(n \cdot T)=u((n-1) \cdot T)+\Delta \tilde{u}(n \cdot T) \\
& \text { and } \\
& \Delta \tilde{u}(n \cdot T)=\sum_{\Delta \tilde{u}_{i, j}(n \cdot T)}^{(\text {nbr_subs-1) }} \underbrace{\sum_{j=3 \cdot i+2}^{3 \cdot i} \tilde{K}_{j}(\cdot) \cdot e((n-j) \cdot T)}_{i=0} \\
& +\Theta(e(\cdot))
\end{aligned}
$$

The expression (24) represents an association of a collection of nbr_subs $\in \mathbb{N}^{+}$linear-PID subsystems as summarized by figure 4 . The following three-input variables

$$
\{e((n-3 \cdot i) \cdot T), e((n-(3 \cdot i+1)) \cdot T), e((n-(3 \cdot i+2)) \cdot T)\}, i=0,1,2, \cdots
$$

are used by the discrete-time subsystem PID control in incremental form, where $e(n \cdot T)=y^{r}(n \cdot T)-y(n \cdot T)$, $y^{r}(n \cdot T)$ and $y(n \cdot T)$ are a set-point/reference signal for process output, and the process output at sampling time $n . T$, respectively.

The zero-order component of the combination (similar to Oo-PID (17)), $\Delta \tilde{u}_{i=0, j=1,2,3}(n \cdot T)$, is required since it is the fundamental engine of the control law as showed in figure 4. $\Theta(e(\cdot))$ is the number of terms $e((n-j) \cdot T)$ not taken into effect during the association (formu lation) of elementary controllers $\Delta \tilde{u}_{i, j}$. 


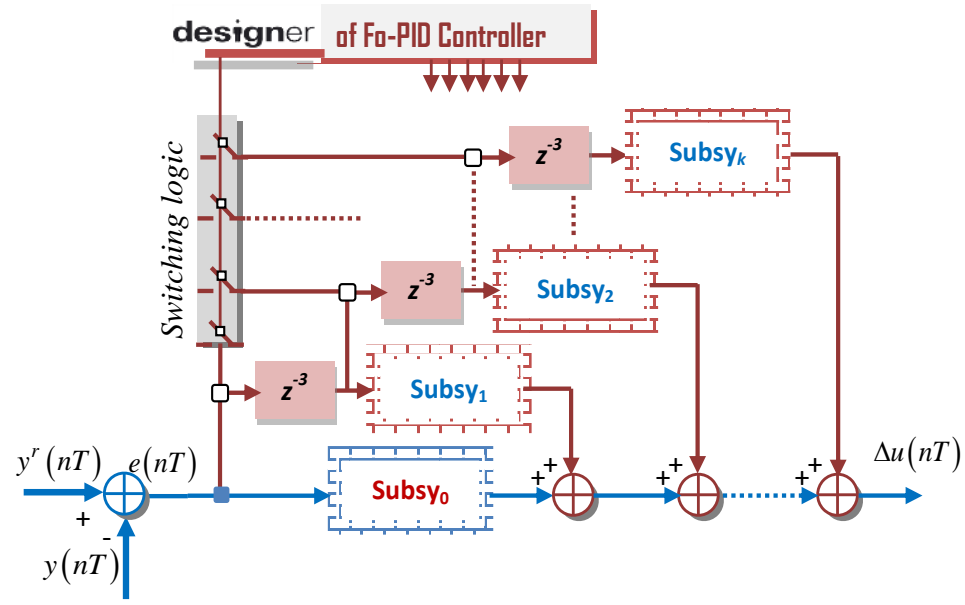

Fig.4. Equivalent model of the developed FoC.

We can consider that the control variation of the newly model developed by (24) will be divided into two parts (B) and (A). Part (B) is the basic element of the control law. Whereas, the additive portion (A) is adjusted to obtain more performances. The control variation (24) can be written in iterative form as:

$$
\begin{gathered}
\Delta \tilde{u}(n \cdot T) \cong \underbrace{\Delta \tilde{u}_{\text {base }}(n \cdot T)}_{(\mathrm{B})}+\underbrace{\sum_{i=1}^{\mathrm{N}} \Delta \tilde{u}_{i, j}(n \cdot T)}_{(\mathrm{A})} \\
\left\{\begin{array}{r}
\Delta \tilde{u}_{i, j}(n \cdot T)=\sum_{j=3 \cdot i}^{3 \cdot i+2} \tilde{K}_{j}(\cdot) \cdot e((n-j) \cdot T) \\
\tilde{K}_{j}(\cdot)=K_{I} \cdot T^{\lambda} \cdot d_{j}+K_{D} \cdot T^{-\mu} \cdot q_{j}
\end{array}\right.
\end{gathered}
$$

where $\quad N \mapsto($ nbr_subs-1) is the upper limit of summation (A). The number of linear regulators $\Delta \tilde{u}_{i, j}(n \cdot T),\{(j=3 \cdot i, 3 \cdot i+1,3 \cdot i+1),(i=1,2, \cdots, N)\}$ in Eq. (25) depends on the number of samples $N$ (temporal universe of operation of the process under study). The maximum number of subsystems in (25) is approximated by

$$
\text { nbr_subs } \leq[N / 3]
$$

where $[N / 3]=\operatorname{int}(N / 3)$ is a means the integer part of $N / 3$. The high order (higher dimension) of the last $i^{\text {th }}$ term in (25), i.e, $j \in[3 \cdot i+2, N]$ be defined by:

$$
\operatorname{order}_{i} \leq[(N-2) / 3]
$$

In this work, the choice of number of subsystems $\Delta \tilde{u}_{i, j}(n \cdot T)$ depends on the desired accuracy and the specifications of the system to be controlled (fast, very fast, slow, etc.) and the monitoring PLC used to implement the algorithm (25).

For slow systems where $T \gg$, needs to be, preferably, a large number of subsystems with respect to the amount of memory reserved for the application. Indeed, for faster systems where $T \ll$, we need the minimum number of selected subsystems must meet a compromise between efficiency and precision of the system to be controlled. From the incremental algorith $m$ of Fo-PID (25), various architectures have been investigated to determine which one will provide the best results in terms of computational speed and accuracy.

- Full Form of Fractional-order PID controller (F2Fo-PID) well suited to slow systems $(T>>)$ ). It requires large space memory (RAM) and significant time- response.

- Extended Form of Oo-PIDC or just Reduced Form of Fractional-order PID controller (EFOo-PIDC or R2Fo-PIDC), where $R_{L}{ }^{\infty}(e(\cdot)) \mapsto 0$ is well suited to very fast systems $(T<<)$. It requires a short storage space and much reduced timeresponse.

- Optimal Form of Fractional-order PID (O2FoPIDC) that represents the F2Fo-PIDC with an optimal number of EFOo-PID subsystems. The value of nbr_subs in (25) is selected and adjusted, by application of learning algorith ms to search the optimal value, to achieve the control design specifications with respect to the functional characteristics of the plant to be controlled. The O2Fo-PID model represented a trade-off between simplicity and efficiency. Moreover, it requires a reduced storage space and optimal time-response.

The R2Fo-PID and O2Fo-PID approaches make the simulation and implementation of Fo-PIDC much easier and enables a smooth transaction for industry to take advantages of this new approach. Further, the effectiveness of the proposed controllers remains to be approved through application examples in different industrial and scientific disciplines. 


\section{Stability analysis of the proposed Fo-PID controller:} An overview

The study of the stability of fractional-order systems can be carried out by studying the solutions of the differential equations that characterize them, linear or nonlinear. Every study of stability of linear time invariant fractional-order (LTI-Fo) system is, generally, based on frequency-domain analysis. An alternative way is the study of the transfer function of the system [23]. There are even some attempts to develop polynomial techniques, either Routh or Jury type, to analy ze their stability [10], [68, 69]. Saeed Balochian et al. [70] present the stabilization problem of a LTI-Fo switched system by a single Lyapunov function whose derivative is negative and bounded by a quadratic function within the activation of each subsystem. The switching law is extracted based on the variable structure control with a sliding sector.

Stability of the fractional-order nonlinear system is very complex and is different from the fractional-order linear systems. The main difference is that for a nonlinear system, it is necessary to investigate steady states having two characteristics, such as limit cycle and equilibrium point. For nonlinear systems (may have several equilibrium points), there are many definitions of stability (asymptotic, global, orbital, ...etc.) [10]. Stability of fractional-order nonlinear dynamic systems is studied in [71] by using Lyapunov direct method with the introduction of Mittag-Leffler stability and generalized Mittag-Leffler stability notions. Actually, in the field of fractional-order control, many stability methods are based on the original Matignon's stability theorem and the classic Lyapunov stability theory. Recent examples include:

$\checkmark \quad$ Fractional-order linear matrix inequalities method $[72,73]$,

$\checkmark \quad$ The robust interval check method [74];

$\checkmark \quad$ Fractional-order Lyapunov inequality method [71].

In this subsection, by using the small gain theorem (SGT) [75] (see appendix), we will find the generalized sufficient BIBO stability conditions of the O2Fo-PID control system (25). Figure 5, simplified form of figure 2 well suited to the analysis of the BIBO stability, shows a general nonlinear control system in a block diagram form. The subsystem $f$ is a fractional controller model and $g$ is a nonlinear dynamic system to be controlled. It is clear from figure 5 that:

$$
\left\{\begin{array} { c } 
{ e _ { 1 } ( t ) = u _ { 1 } ( t ) - \overbrace { g ( e _ { 2 } ( t ) ) } ^ { y _ { 2 } ( t ) } } \\
{ e _ { 2 } ( t ) = u _ { 2 } ( t ) + \underbrace { f ( e _ { 1 } ( t ) ) } _ { y _ { 1 } ( t ) } }
\end{array} \text { or } \left\{\begin{array}{l}
u_{1}(t)=e_{1}(t)+\overbrace{g\left(e_{2}(t)\right)}^{y_{2}(t)} \\
u_{2}(t)=e_{2}(t)-\underbrace{f\left(e_{1}(t)\right)}_{y_{1}(t)}
\end{array}\right.\right.
$$

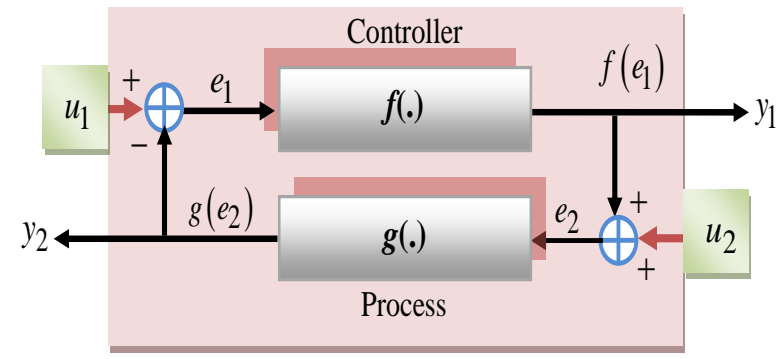

Fig.5. A typical closed-loop control system.

Suppose that the incremental control formula of fractional control law (25) is used. By defining

$$
\left\{\begin{array}{l}
e_{1}(n \cdot T)=e(n \cdot T), \quad e_{2}(n \cdot T)=u(n \cdot T) \\
u_{1}(n \cdot T)=y^{r}(n \cdot T), \quad u_{2}(n \cdot T)=u((n-1) \cdot T) \\
\Delta u(n \cdot T)=f(e(n \cdot T)), \quad y(n \cdot T)=g(u(n \cdot T))
\end{array}\right.
$$

It's easy to see that we obtain an equivalent closedloop control system as shown in figure 5, we have from (28)

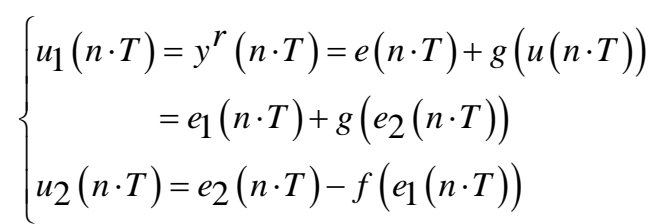

Based on the general form of the incremental $\mathrm{O} 2 \mathrm{Fo}$ PID form (25), we have

$$
\begin{aligned}
\|\Delta u(n \cdot T)\|=\mid \Delta u_{\text {base }}(n \cdot T)+\sum_{i=1}^{N} \Delta \tilde{u}_{i, j}(n \cdot T) \| \\
\quad \leq\left|\Delta u_{\text {base }}(n \cdot T)\right|+\left|\sum_{i=1}^{N} \Delta \tilde{u}_{i, j}(n \cdot T)\right| \\
\quad \leq \underbrace{\left|\tilde{K}_{0}(\cdot)\right|}_{\tilde{\gamma}_{1}}|\cdot| e(n \cdot T) \mid+ \\
+\left|\tilde{K}_{1}(\cdot) \cdot e((n-1) \cdot T)+\tilde{K}_{2}(\cdot) \cdot e((n-2) \cdot T)\right| \\
+\left|\sum_{i=1}^{N} \Delta \tilde{u}_{i, j}(n \cdot T)\right|
\end{aligned}
$$

where

$$
i=1, \cdots, N ; j=\{3,4,5\},\{6,7,8\}, \cdots,\{n-2, n-1, n-3\}
$$

and

$$
\tilde{\gamma}_{1}=\left|K_{P}+K_{I} \cdot T^{\lambda}+K_{D} \cdot T^{-\mu}\right|
$$


and

$$
\begin{aligned}
\|y(n \cdot T)\| & =\left\|g\left(e_{2}(n \cdot T)\right)\right\|=\|g(u(n \cdot T))\| \\
& \leq\|g\| \cdot|u(n \cdot T)|
\end{aligned}
$$

which is of the form

$$
\tilde{\gamma}_{2}=\|g\|<\infty
$$

The operator norm $\|g\|$ is the gain of the given nonlinear system $g(\cdot)$, usually defined as [76]

$$
\|g\|=\sup _{u_{1} \neq u_{2}, n \geq 0} \frac{\left|g\left(u_{1}(n)\right)-g\left(u_{2}(n)\right)\right|}{\left|u_{1}(n)-u_{2}(n)\right|}
$$

The norm is the gain of the given nonlinear system over a set of admissible control signals that have any meaningful function norms and $u_{1}(n)$ and $u_{2}(n)$ are any two of the control signals in the set.

So, the sufficient conditions for the O2Fo-PIDC system to be BIBO stable if the parameters of the O2FoPIDC satisfy the inequality $\tilde{\gamma}_{1} \cdot \tilde{\gamma}_{2}<1$ where $\tilde{\gamma}_{1}$ and $\tilde{\gamma}_{2}$ are defined in (32) and (34), respectively.

Theorem: Sufficient conditions of the O2Fo-PIDC system (25) shown in figure 2 to be globally BIBO stable are

$i$. The given nonlinear process has a bounded norm, i.e., the nonlinear system has a finite gain $\{\|g\|<+\infty\}$ and

ii. The knowledge base of the O2Fo-PIDC $\left\{\left\{K_{P}, K_{I}, K_{D}\right\},\{\lambda, \mu\},\{T\}\right\}$ satisfies the condition $\left(\left|K_{P}+K_{I} \cdot T^{\lambda}+K_{D} \cdot T^{-\mu}\right|\right) \cdot\|g\|<1$.

\section{Conditions:}

$$
\left\{(\overbrace{\mid K_{P}+K_{I} \cdot T^{\lambda}+K_{D} \cdot T^{-\mu}}^{\tilde{\gamma}_{1}}) \cdot \begin{array}{l}
\|g\|<1 \text { and } \\
\tilde{\tilde{\gamma}_{2}}
\end{array}\right.
$$

together provide the $\mathrm{BIBO}$ stability criteria for the $\mathrm{O} 2 \mathrm{Fo}$ PIDC design for a given bounded system. By noting that $K_{P}>0, K_{I}>0, K_{D}>0, T>0$, the result for the stability of $\mathrm{O} 2 \mathrm{Fo}-\mathrm{PIDC}$ system will be:

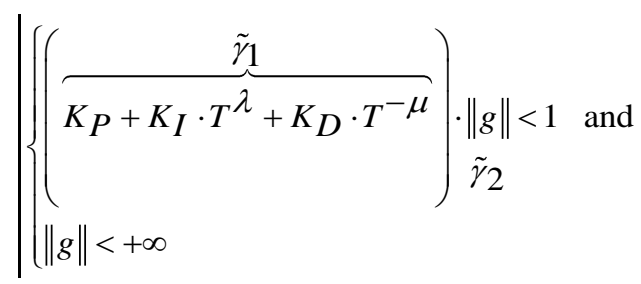

Applying the SGT, we can obtain the sufficient conditions for the BIBO stability for the linear Oo-PIDC system, where $\lambda=1$ and $\mu=1$ in (37).

It can be remarked that the stability conditions for the controllers O2Fo-PID, R2Fo-PID and F2Fo-PID are similar.

The zero-order component of the combination, $\Delta \tilde{u}_{i=0, j=1,2,3}(n \cdot T)$, is required in study of stability analysis, since it is the fundamental engine of the control law. The other terms of the combination may affected, either locally or globally, on the precision, robustness and the possibility of the real-time implementation of the control law (25).

\section{OPTIMIZATION OF THE O2FO-PID CONT ROLLER}

Multiobjective optimization (MO) process is applied which simultaneously minimizes $n$ objective functions $J(\vartheta)$ which are functions of decision variables $\vartheta$ bounded by some equality and inequality constraints (constraints could be linear or nonlinear).

A MO problem can be formulated as follows

$$
\text { Minimizes } \underset{\substack{J(\vartheta) \\ \vartheta \in \mathbb{R}}}{J\left(J_{1}(\vartheta), \ldots, J_{k}(\vartheta)\right]^{T}}
$$$$
\text { Subject to }
$$$$
\begin{aligned}
& \mid \begin{array}{l}
\text { Functional constraints } \\
g_{j}(\vartheta) \leq 0, \\
h_{i}(\vartheta)=0, \quad j \in[1, l]
\end{array} \\
& \mid \begin{array}{l}
\text { Parametric constraints } \\
\theta_{r} \leq \vartheta_{r} \leq \overline{\theta_{r}}, \quad r \in[1, n]
\end{array}
\end{aligned}
$$

where $\vartheta \in \mathbb{R}$ is defined as the decision vector, $J(\vartheta) \in \mathbb{R}^{k} \quad$ as the objective function vector. $g(\vartheta)=\left\{g_{j}(\vartheta)\right\},(j=1,2, \ldots, l)$ and $h(\vartheta)=\left\{h_{i}(\vartheta)\right\},(i=1,2, \ldots, m)$ are the vector of equality and inequality constraints, respectively. $\theta_{r}$ and $\overline{\theta_{r}}$ are the lower and upper bounds in the decision space for $\vartheta_{r}$ parameter. The minimization problem (38) can be further represented as 


$$
\begin{array}{r}
\text { Minimizes } G(\vartheta) \\
\vartheta \in \mathbb{R}
\end{array}
$$

where $G(\vartheta)$ incorporates the objective function $J(\vartheta)$ and the constraint functions $g(\vartheta)$ and $h(\vartheta)$, respectively. There are different ways to convert (38) into (39), i.e., the transformation of the optimization problem (38) to an unconstrained problem is made through many methods. The weighted sum (or min-max) of objectivesconstraints methods are the two popular approaches [77]. In order to incorporate constraints into the optimization problem, the sequential unconstrained minimization technique is used. Here, the pseudo objective function is formulated as

$$
\underset{\vartheta \in \mathbb{R}}{G}(\vartheta, r \mathrm{P})=\underset{\vartheta \in \mathbb{R}}{\tilde{G}}(\vartheta)+r_{\mathrm{P}} \cdot \underset{\vartheta \in \mathbb{R}}{\Pi}(\vartheta)
$$

$\tilde{G}_{(\vartheta)}$ is a combination of objectives in (38) (sum or $\vartheta \in \mathbb{R}$

max of objectives) and $\prod_{\vartheta \in \mathbb{R}}(\vartheta)$ is termed the penalty function that introduces the constraints in (38) into the cost function. $r$ denotes the scalar penalty multiplier and is typically increased as the optimization goes on to put more and more emphasis on avoiding constraint violations [78]. Metaheuristic algorithms, that include
GA, are rapidly becoming the most used methods of choice for some intractable systems [79-81].

\section{A. Genetic optimization of the developed FoC}

GA are global parallel search and optimization techniques based around Darwinian principles, working on a population of potential solutions (chromosomes). Every individual in the population represents a particular solution to the problem under study, often expressed in binary (or real) code. The population is evolved over a series of generation (based on selection, crossover and mutation operators) to produce better solutions to the problem.

Figure 6 shows the structure of the control design and optimization process for a nonlinear dynamical system that contains three main blocks

$\checkmark$ Optimization layer characterized by the MGA;

$\checkmark$ Control layer representing the closed loop O2FoPIDC strategy;

$\checkmark \quad$ Dynamical systemto be controlled.

Once the 'optimal' structure of the controller is identified as given in (25), the optimization of the O2FoPID model is to find the 'best' parameters, i.e., an optimal knowledge base, which can be represented as an extremum problem of optimization index (39).

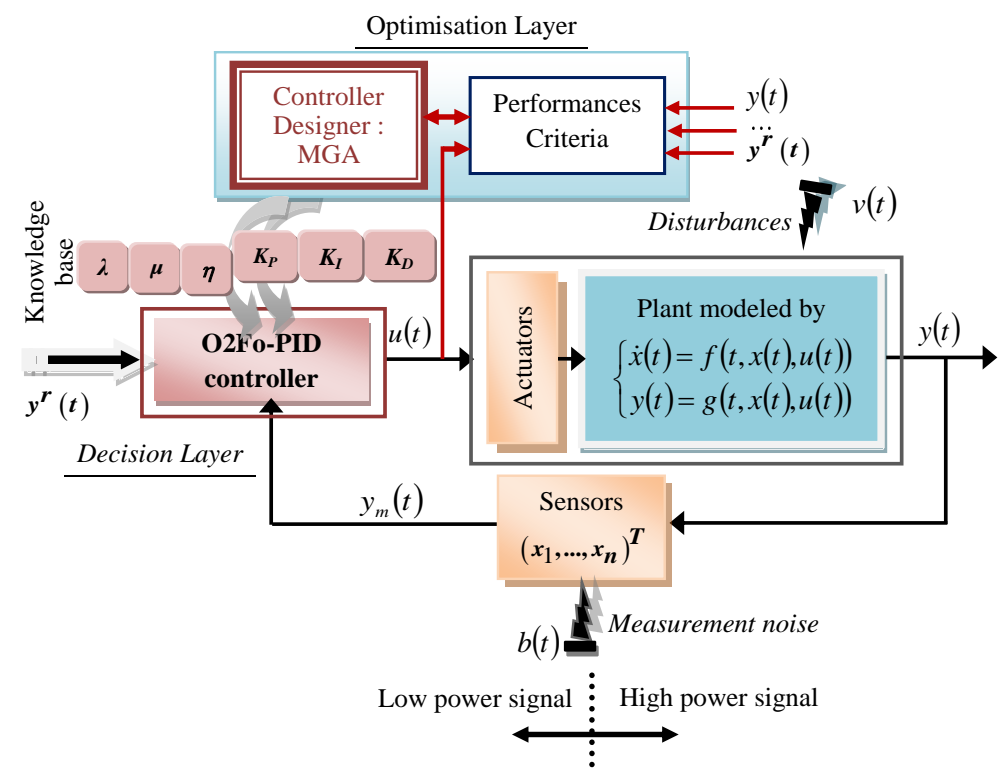

Fig.6. Elements of the control loop.

There are many methods to design such fractional controllers which can be regarded as some optimization problems of certain performance measures of the controlled systems.

The controller design based on GA can be considered as a MO search procedure over a large objectiveparameter space. Figure 7 summarizes the evolution process of GA, used in this work, to design the optimal FoC able to realize the desired objectives.
Using arith metic map and specified probability do crossover of parents to form new offsprings Adaptation of genetic operators to appropriate coding mode-.

$\checkmark$ Using chaotic map and specified probability mutate chro mosomes to form new offs pring's. The chaotic mutation operator [82] is used to solve the problem of maintaining the population diversity of $\mathrm{GA}$ in the learning process. 
The concept of elite strategy is adopted, where the elites - Improvement of the basic algorithm -. best individuals in a population are regarded as

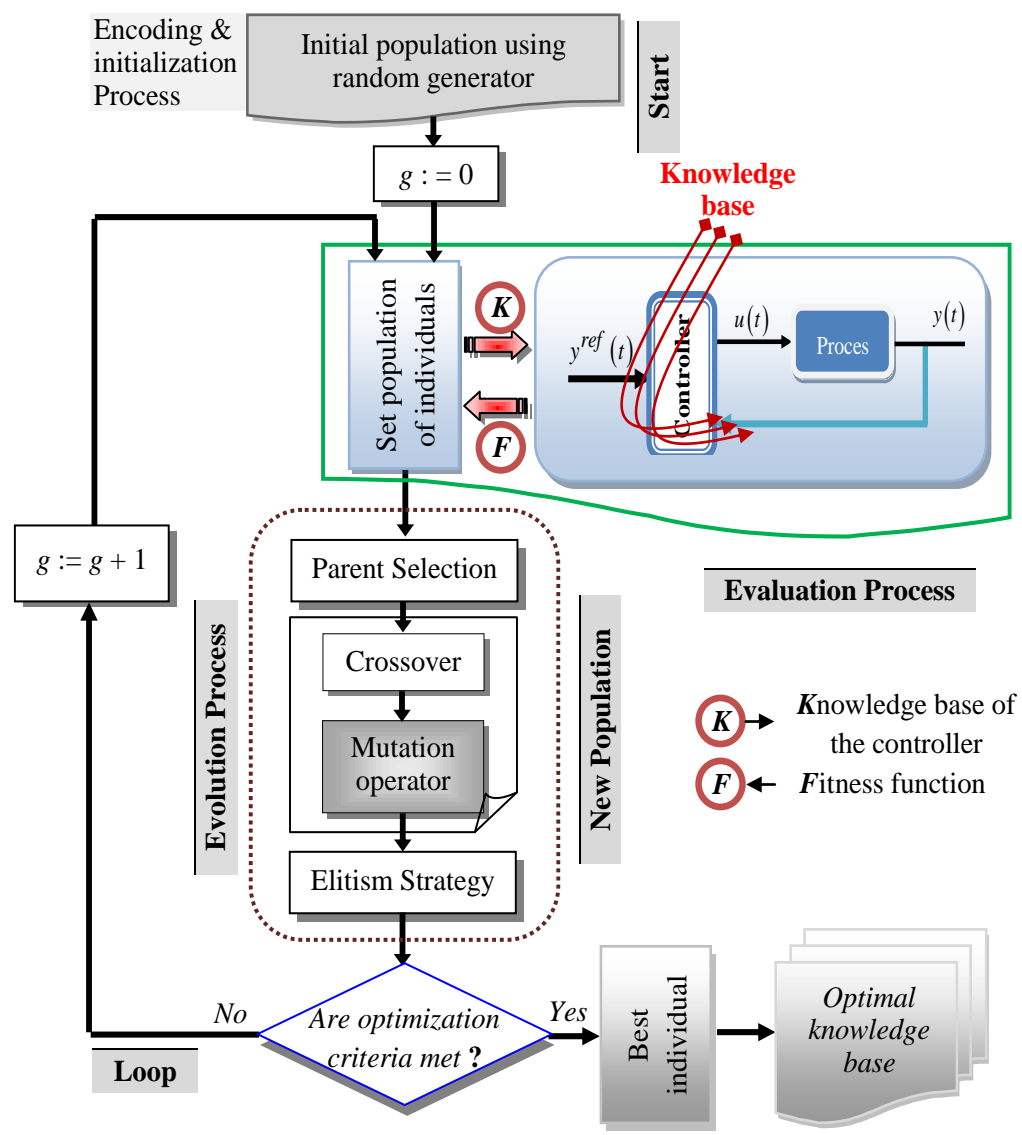

Fig.7. A general cycle of the GA and the interaction between GA-closed loop control systems.

The parameters to be optimized are obviously the O2Fo-PIDC gains, non-integer integrator-differentiator orders and the number of PID subsystems. So, the optimized parameter set may be such that

$$
\begin{aligned}
\tilde{K}_{b}= & {\left[\begin{array}{ll}
\tilde{G}_{c} & \tilde{S}_{c}
\end{array}\right]^{T}, } \\
& \left\{\begin{array}{lll}
\tilde{G}_{c}=\left[\begin{array}{lll}
K_{P} & K_{I} & K_{D}
\end{array}\right]^{T}, \\
\tilde{S}_{c}=\left[\begin{array}{lll}
\lambda & \mu & \text { nbr_subs }
\end{array}\right]^{T}
\end{array}\right.
\end{aligned}
$$

Therefore, three different possibilities exist in general

$$
\begin{array}{lll}
\checkmark & \text { Optimize gains only } \tilde{G}_{c} ; \\
\checkmark & \text { Optimize order only } \tilde{S}_{c} ; \\
\checkmark & \text { Optimize all parameters } \tilde{K}_{b} .
\end{array}
$$

In order to employ the GA to optimize the knowledge base of the FoC, we establish the fitness function according to the objectives specified by the designer. Thus, the controller design based on GA can be considered as a MO search procedure over a large objective-parameter space. $\checkmark$ Small maximum error with small total squared error $\left(J_{1}\right)$;

$\checkmark$ Reduced control effort to be applied to the process $\left(J_{2}\right)$;

$\checkmark$ Reduced number of linear-PID subsystems $\left(J_{3}\right)$;

$\checkmark$ Stability analysis conditions $\left(g_{s t}\right)$.

The objectives-constraints for a MIMO system with $m$ outputs are given by their expressions:

$$
\begin{aligned}
& \left\{\begin{aligned}
J_{1}(\tilde{x}(k)) & =\frac{1}{M} \cdot \sum_{k=0}^{N-1} \tilde{x}^{T}(k) \cdot Q(k) \cdot \tilde{x}(k) \\
& +\frac{1}{M} \cdot \max _{k}\left\{\tilde{x}^{T}(k) \cdot Q(k) \cdot \tilde{x}(k)\right\} \\
Q(k) \quad & \beta_{1}(k) \cdot \mathrm{I}_{n \times n}
\end{aligned}\right. \\
& \left\{\begin{array}{l}
J_{2}(u(k))=M^{-1} \cdot \sum_{k=0}^{N-1} u^{T}(k) \cdot R(k) \cdot u(k) \\
R(k) \quad=\beta_{2}(k) \cdot \mathrm{I}_{m \times m}
\end{array}\right.
\end{aligned}
$$




$$
\begin{gathered}
\left\{\begin{array}{l}
J_{3}(\text { nbr_subs })=\left\{\eta_{\mathrm{sub}}^{T} \cdot S(i) \cdot \eta_{\mathrm{sub}}\right\} \\
S(i) \quad=\beta_{3}(i) \cdot \mathrm{I}_{m \times m}
\end{array}\right. \\
\left\{\begin{array}{l}
\tilde{g}(\theta)=\frac{1}{m} \cdot g_{s t}^{T}(\theta) H(i) \cdot g_{s t}(\theta) \\
H(i)=\beta_{3}(i) \cdot \mathrm{I}_{m \times m} \\
\underbrace{g_{s t}(\theta)_{i}}_{i=1, \cdots, m}=\left(K_{P i}+K_{I i} \cdot T^{\lambda i}+K_{D i} \cdot T^{-\mu}\right) \cdot\|g\|-1<0
\end{array}\right.
\end{gathered}
$$

$M=[N / T]$ denotes the integer number of computing steps $N$ is the running time and $T$ is the sampling period. $\tilde{x}(k)=x^{r}(k)-x(k)$ is the tracking error at sampling time $k . \mathrm{I}_{l \times m}$ is the identity matrix of dimension $l \times m$ and $n$ is the number of state variables. $\eta_{\text {sub }}$ is a vector of linear-PID subsystems. The weighting factors $\beta_{j}(\cdot)>0,(j=1,2,3)$, selected to provide a compromise between design specifications.

By using the interior penalty function (IPF) method, the stability constraints are included. The transformed unconstrained problem via the IPF method, used in this work, is given by [78]

$$
\text { Minimizes } \underset{\vartheta \in \mathbb{R}}{G(\vartheta)}=\sum_{j=1}^{n} w_{i} \cdot J_{i}(\vartheta)+\prod_{\vartheta \in \mathbb{R}}\left(\vartheta, r_{g}, r_{h}\right)
$$

where $\prod_{\vartheta \in \mathbb{R}}\left(\vartheta, r_{g}, r_{h}\right)$ is the IPF, expressed as

$$
\begin{aligned}
\prod_{\vartheta \in \mathbb{R}}\left(\vartheta, r_{g}, r_{h}\right)=r_{h} & \cdot\left[\sum_{k=1}^{l} h_{k}(\vartheta)^{2}\right] \\
+r_{g} & \cdot\left[\sum_{j=1}^{m} \frac{-1}{g_{j}(\vartheta)}\right]
\end{aligned}
$$

In the expression (46), $n=3$ and $h_{k}(\vartheta)=0, \forall k \in \mathbb{N}$. The function $G(\vartheta)$ in (46), mainly, depends on the design specifications required by the user. The weights $\left(w_{i}, r_{g}\right)>0$ can, therefore, be used in control system design as design parameters to trade-off between different performance specifications. From (46), the local and global minima can be calculated if the region of realizability of $\vartheta$ is convex [77]. It is usually assumed that

$$
\sum_{i}\left(w_{i}, r_{g}, r_{h}\right)=1
$$

The expression (48) is a compromise between better accuracy, a reduced consumption of energy control (more security), optimal structure and satisfied stability analysis conditions. In the rest of the algorith $\mathrm{m}$, the performance criterion to minimize, will be selected and adapted to the case of MIMO systems. The additional constraints are the lower $\underline{\theta}=\underline{K}_{b}^{T}$ and upper $\bar{\theta}=\bar{K}_{b}^{T}$ limits of the controller parameters. The optimization procedure may be pronounced as follows: Find the knowledge base $\tilde{K}_{b}^{T}$ (41) that

$$
\begin{array}{ll}
\text { Minimizes } & G(\vartheta) \\
& \vartheta \in \mathbb{R} \\
\text { Subject to } & \\
& \underline{\theta} \leq \theta \leq \bar{\theta}
\end{array}
$$

The fitness function is a measure of how well a set of candidate coefficients meet the design specifications. A fitness value used by the MGA can then defined as

$$
\operatorname{Fitness}_{\vartheta \in \mathbb{R}}(\vartheta)=\frac{\delta}{\xi+G(\vartheta)}
$$

where $(\xi, \delta) \in \mathbb{R}^{2}$ and $\xi$ is a s mall positive constant used to avoid the numerical error of dividing by zero. Four major operations are necessary for correctly handling the execution of the optimization algorithm:

$\checkmark$ Determine the O2Fo-PID knowledge base to be optimized;

$\checkmark$ Determine an optimization objective;

$\checkmark$ Constraint equations:

$\checkmark$ Bounds for the elements of the design vector.

MGA based learning provide an alternative way to learn for the O2Fo-PID knowledge base $\tilde{K}_{b}(41)$. The adaptation of these parameters continues until the overall number of generations is satisfied.

\section{B. Chromosome structure}

The key to put a genetic search for the O2Fo-PIDC into practice is that all design variables to be optimized (41) are encoded as a finite length string, called chromosome.

The real-valued (or mixed) genes representation is used in this work. Global structure of the chromosome, in the case of MIMO systems, and the corresponding configurations is illustrated in figure 8 .

Every chro mosome which encodes the knowledge base of the O2Fo-PIDC can represent a solution of the problem, that is, a O2Fo-PID optimal knowledge base. To simplify the genes coding mode, i.e., avoidance of the mixed coding (real-integer) and the genetic operators adapted for each mode, the real-coding is used with the 
restriction

$$
\begin{gathered}
\text { nbr_subs }=\left[\eta_{c t r l}\right] \in \mathbb{N}^{+} \\
\left\{\begin{array}{l}
\eta_{c t r l} \in \mathbb{R}^{+} \\
\eta_{c t r l} \in[1,[N / 3]]
\end{array}\right.
\end{gathered}
$$

where $\eta_{c t r l}$ models the number of subsystems, nbr_subs $\in \mathbb{N}^{+}$, included in modeling of O2Fo-PIDC control law under development of (25) and $[x]$ represents the integer part of $x$.
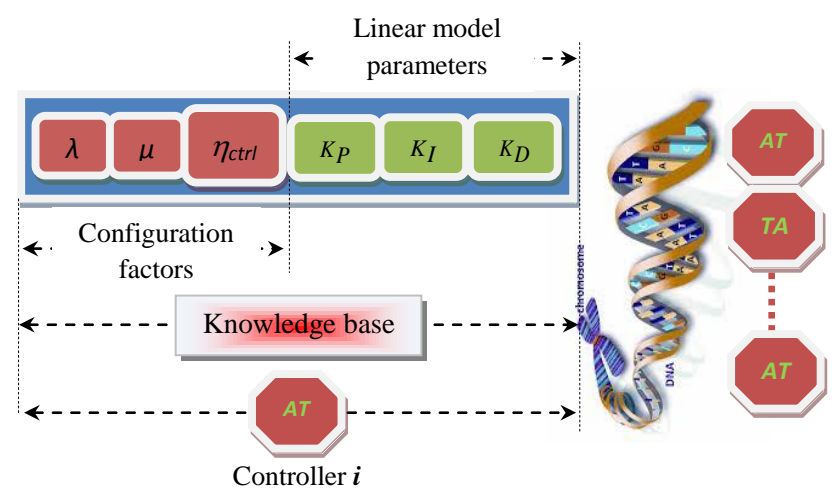

(a)

(b)

Fig.8. Chromosome structure of O2Fo-PID controller for (a) SISO systems. (b) MIMO systems.

In the next section, the developed algorithm is applied to stabilize the CE150 helicopter model. The process is modelled by a strongly nonlinear MIMO system.

\section{Simulation}

The proposed design controller is applicable to a specific class of nonlinear system that can be described by the differential equation of form

$$
\begin{aligned}
& \frac{d x(t)}{d t}=\tilde{f}(t, x(t), u(t), v(t)) \\
& y(t)=\tilde{g}(t, x(t), u(t), v(t)) \\
& \left\{\begin{array}{l}
\frac{d v(t)}{d t}=s(v(t)) \\
x(o)=x_{0}, \quad t \in\left[0, t_{f}\right]
\end{array}\right.
\end{aligned}
$$

where $x(t) \in \mathbb{R}^{n}$ and $u(t) \in \mathbb{R}^{m}$ are the state vector and the control vector, respectively. $v(t) \in \mathbb{R}^{q}$ represents the state of an external signal generator (dynamic of disturbances), $x_{0}$ is the initial state vector, $\tilde{f}: C^{\infty}\left(\mathbb{R}^{n} \times \mathbb{R}^{m}\right) \rightarrow \mathbb{R}^{n}$ is a nonlinear relationship between the state variables of the controlled system, the generated control signals and interference (disturbances) signals, respectively. The output or observation $y(t) \in \mathbb{R}^{q}$ is generated via an output map $\tilde{g}(\cdot)$.

\section{A. Control of a nonlinearhelicopter process}

The CE150 helicopter model offered by Humusoft Ltd [83] for the theoretical study and practical investigation of basic and advanced control engineering principles. The system consists of a body, carrying two propellers driven by DC motors and a massive support. The body has two degrees of freedom. Both body position angles (horizontal and vertical) are influenced by the rotation of propellers. The axes of a body rotation are perpendicular. Both body position angles, i.e. azimuth angle $\Psi$ in horizontal and elevation angle $\Phi$ in vertical plane are influenced by the rotating propellers, simultaneously. The DC motors for driving propellers are controlled proportionally to the output signal of the computer. All inputs and outputs variables are coupled. The user of the simulator communicates with the system via the dataprocessing interface. The schematic diagram of the helicopter model is shown in figure 9.

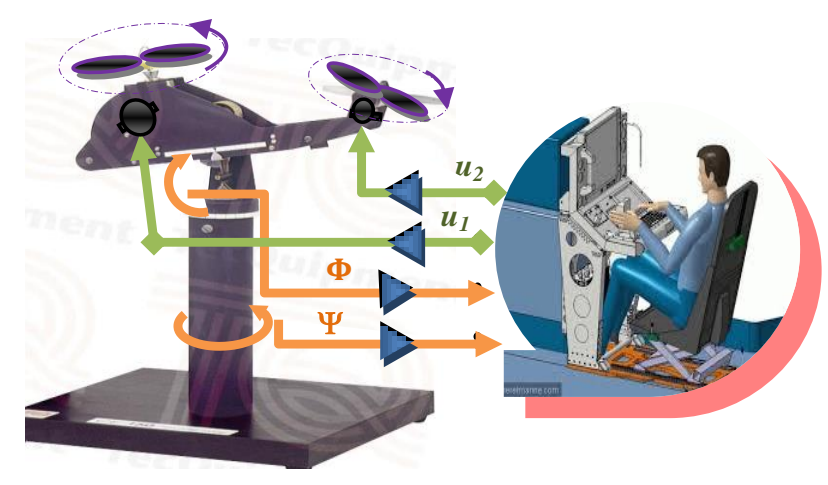

Fig.9. Helicopter simulator configuration.

The helicopter model is a multivariable dynamical system with two manipulated inputs and two measured outputs. The system is essentially nonlinear, naturally unstable with significant cross coupling. The mathematical model of the helicopter is given by the following differential equations system[84] 


$$
\left\{\begin{aligned}
\dot{x}_{1}(t)= & x_{2}(t) \\
\dot{x}_{2}(t)= & 0.8764 \cdot x_{2}(t) \cdot \sin \left(x_{1}(t)\right)+3.4325 \cdot x_{4}(t) \cdot \cos \left(x_{1}(t)\right) \cdot u_{1}(t) \\
+ & 0.4211 \cdot x_{2}(t)-0.0035 \cdot x_{5}{ }^{2}(t)- \\
& 46.35 \cdot x_{6}^{2}(t)-0.8076 \cdot x_{5}(t) \cdot x_{6}(t)-0.0259 \cdot x_{5}(t) \\
& -2.9749 \cdot x_{6}(t) \\
\dot{x}_{3}(t)= & x_{4}(t) \\
\dot{x}_{4}(t)= & 21.4010 \cdot x_{4}(t)-31.8841 \cdot x_{8}{ }^{2}(t)-14.2029 \cdot x_{8}(t) \\
& -21.7150 \cdot x_{9}(t)+1.4010 \cdot u_{1}(t) \\
\dot{x}_{5}(t)= & -6.6667 \cdot x_{5}(t)-2.7778 \cdot x_{6}(t)+2 \cdot u_{1}(t) \\
\dot{x}_{6}(t)= & 4 \cdot x_{5}(t) \\
\dot{x}_{7}(t)= & -8 \cdot x_{7}(t)-4 \cdot x_{8}(t)+2 \cdot u_{2}(t) \\
\dot{x}_{8}(t)= & 4 \cdot x_{7}(t) \\
\dot{x}_{9}(t)= & -1.3333 \cdot x_{9}(t)+0.0625 \cdot u_{1}(t)
\end{aligned}\right.
$$

where, $x_{1}(t)=\Psi(t), x_{2}(t)=d \Psi(t) / d t, x_{3}(t)=\Phi(t)$, $x_{4}(t)=d \Phi(t) / d t$ and $x_{5}(t)-x_{9}(t)$ are state variables representing the two DC motors and the coupling effects. Assuming that the helicopter model (53) is a rigid body with two degrees of freedom, the helicopter simulator CE150 can be viewed as an interconnexion of two subsystems. The elevation subsystem characterized by the input/output variables $\left(u_{1}(t), \Psi(t)\right)$ and the azimuth subsystem characterized by the input/output variables $\left(u_{2}(t), \Phi(t)\right)$, where, $\Psi(t)$ is the elevation angle (pitch angle), $\Phi(t)$ is the azimuth angle (yaw angle), $u_{1}(t)$ is the main motor voltage and $u_{2}(t)$ is the tail motor voltage. The decentralized O2Fo-PIDC strategy is adopted in this application, where the multivariable process is treated as two separate single variables process, namely:

$$
\begin{aligned}
& u_{1}(t)=\mathrm{O}_{2} \mathrm{Fo}_{\mathrm{PID}}(1)\left(\Psi^{r}(t), \Psi(t)\right) \text { and } \\
& u_{2}(t)=\mathrm{O}_{2} \mathrm{Fo}_{\mathrm{PID}}(2)\left(\Phi^{r}(t), \Phi(t)\right)
\end{aligned}
$$

where $\Psi^{r}(t)$ and $\Phi^{r}(t)$ are the desired values (setpoints) of the considered variables. Figure 10 su mmarizes the block diagram of the feedback control by using the developed O2Fo-PIDC.

In our application, the helicopter model is dicretized by using the fourth-order Runge-Kutta method with sampling time chosen to be $0.1[\mathrm{sec}]$ and a time horizon of $50[\mathrm{sec}]$. The control objective is to stabilize the system states $\left[\begin{array}{ll}\Psi(t) & \Phi(t)\end{array}\right]^{T}$ around a set-point $\left[\begin{array}{ll}\Psi^{r}(t) & \left.\Phi^{r}(t)\right]=[1[\mathrm{rad}] \\ 1[\mathrm{rad}]\end{array}\right]^{T}$ under the control actions $\left[\begin{array}{ll}u_{1}(k) & u_{2}(k)\end{array}\right]^{T}$. The initial conditions, used in simulation of the process, are taken as $x(t)=\left[\begin{array}{llll}0 & 0 & \cdots & 0\end{array}\right]^{T}$.

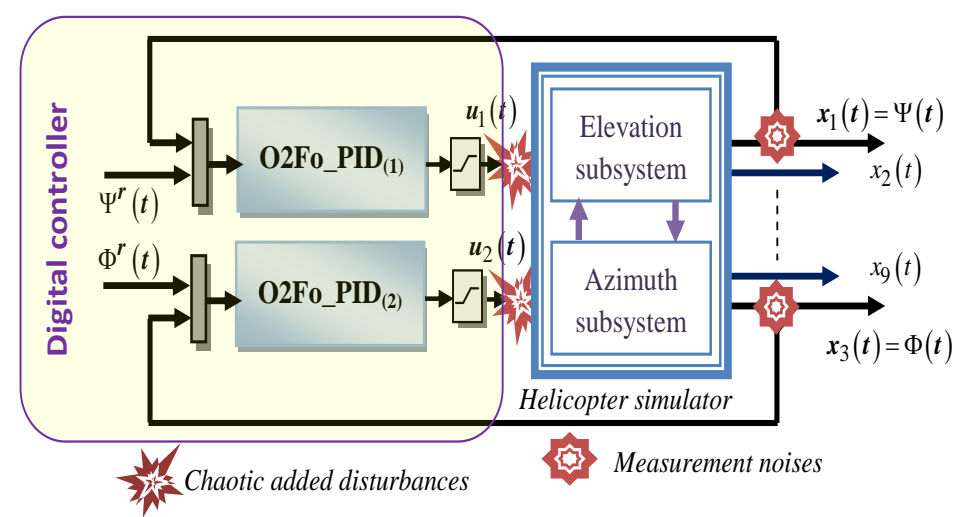

Fig.10. Block diagram of the feedback control of the Helicopter model using two O2Fo-PIDC. 
The disturbances used to test a particular control strategy play a critical evaluation role. Thus, to carry out a complete and unbiased evaluation, it is necessary to define a series of disturbances and to subject each control strategy to all disturbances.

Chaotic wave perturbations [83] on the system states and the controller signals are added in order to tes the efficiency and robustness of the controller.

\section{B. Simulation results}

The adapted chromosome structure with real-coding of genes of the MGA for this application has the same form of the figure 8 (a), but doubled. A part for $\mathrm{O}_{2} \mathrm{Fo}-\mathrm{PID}_{(1)}$ and the other for $\mathrm{O} 2 \mathrm{Fo}-\mathrm{PID}_{(2)}$. The MGA characteristics are summarized in table 1.

Figures 11 (a)-(d), show, respectively, the evolution of the control parameters $K_{P i}, K_{I i}, K_{D i}, \lambda_{i}, \mu_{i} \quad(i=1,2)$ of the controllers O2Fo-PID $(1)$ and O2Fo-PID $(2)$ during the optimization process. Around 500 optimization iteration steps (generation number), the number of linear-PID subsystems (51) and the objective function (49) are minimized as shown in figures 12 (a) and (b), respectively. It can be remarked that the nu mber of linear PID subsystems is minimized to $80 \%$ of its maximum value.

For generalization, a comparison of the evaluation values obtained at the end of the algorithm execution between the proposed O2Fo-PIDC (25), the classical OoPIDC, F2Fo-PIDC and R2Fo-PIDC using the same optimization procedure (49) is summarized in Table 2.

Table 1. Specifications of the MGA.

\begin{tabular}{ll}
\hline Characteristic & Value \\
\hline Population Size & 50 \\
Max_gen & 500 \\
Coding chromosome & Real \\
Gain factors $K_{P i}, K_{I i}, K_{D i}(i=1,2)$ & {$\left[10^{-6}, 0.1\right]$} \\
Control factors $\lambda_{i}, \mu_{i}(i=1,2)$ & {$\left[10^{-4}, 1.0\right]$} \\
Number of subsystems $\eta_{c t r l 1-2}$ & {$[1.0,166.0]$} \\
Selection process & Tournament \\
Arithmetic Crossover & $P_{c}=0.8$ \\
Chaotic Mutation & $P_{m}=0.02$ \\
\hline
\end{tabular}

As shown in numerical values in Table 2 (rows and columns have been ticked), it has been demonstrated that the newly developed O2Fo-PIDC presents better performances than the R2Fo-PIDC, F2Fo-PIDC and the Oo-PIDC with similar optimization algorithm.

The simulation results at the end of the execution of the algorithm developed in this work are shown schematically in figures 13 and 14 .

Graphical comparative results between O2Fo-PIDC and the Oo-PIDC, F2Fo-PIDC and R2Fo-PIDC are mentioned in these figures. Figures 13 (a)-(b) illustrate the evolution of the output variables and their corresponding reference trajectories.

It has been demonstrated that the developed $\mathrm{O} 2 \mathrm{Fo}-$ PIDC presents better performances then the others controllers with similar optimization algorithm (tuning and stability conditions). The outputs of the developed controllers are illustrated by the figures 14 (a)-(b).

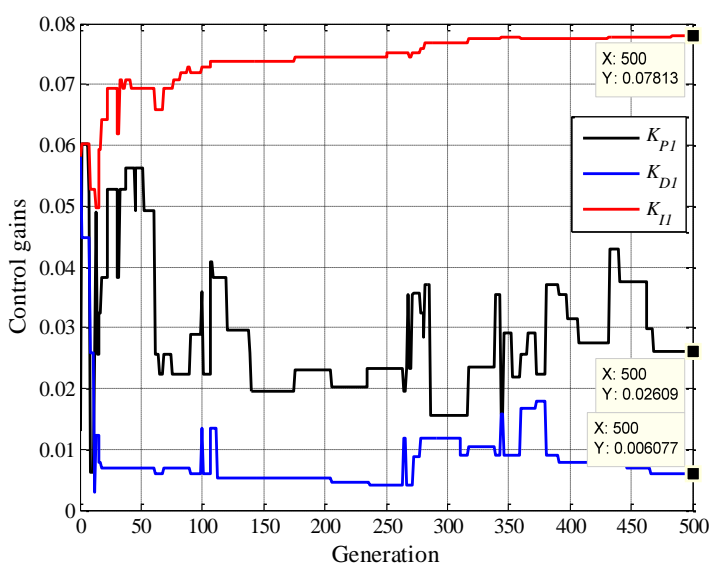

(a)

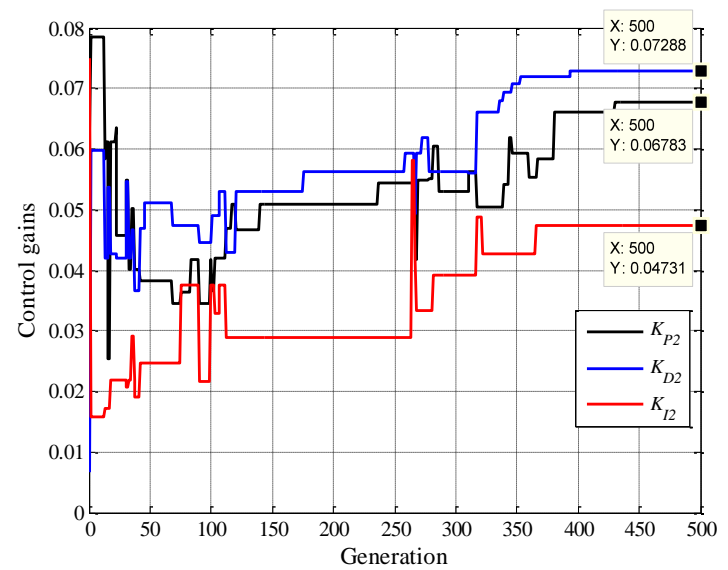

(b) 
Table 2. O2Fo-PIDC, R2Fo-PIDC, F2Fo-PIDC and Oo-PIDC performances.

\begin{tabular}{|c|c|c|c|c|c|c|c|c|}
\hline \multirow{2}{*}{ Parameteê } & \multicolumn{2}{|c|}{ O2Fo-PID } & \multicolumn{2}{|c|}{ R2Fo-PID } & \multicolumn{2}{|c|}{ F2Fo-PID } & \multicolumn{2}{|c|}{ Oo-PID } \\
\hline & $O 2 F o-P I D_{(l)}$ & $O 2 F o-P I D_{(2)}$ & $R 2 F o-P I D_{(l)}$ & $R 2 F o-P I D_{(2)}$ & $F 2 F o-P I D_{(l)}$ & $F 2 F o-P I D_{(2)}$ & $O o-P I D_{(1)}$ & $O o-P I D_{(2)}$ \\
\hline$\overline{K P}$ & 0.026086 & 0.067830 & 0.012292 & 0.060458 & 0.017621 & 0.075926 & 0.010315 & 0.079464 \\
\hline$K_{D}$ & 0.006076 & 0.072876 & 0.007261 & 0.038959 & 0.017838 & 0.077685 & 0.016022 & 0.007824 \\
\hline$K_{I}$ & 0.078126 & 0.047305 & 0.074661 & 0.063871 & 0.079673 & 0.023698 & 0.078145 & 0.008156 \\
\hline$\lambda$ & 0.613409 & 0.555782 & 0.613581 & 0.247233 & 0.991755 & 0.851211 & 1 & 1 \\
\hline$\mu$ & 0.027912 & 0.493221 & 0.011761 & 0.171282 & 0.855631 & 0.352196 & 1 & 1 \\
\hline nbr_subs & $1_{\text {base }}+8$ & $1_{\text {base }}+8$ & $\mathbf{1}_{\text {base }}+0$ & $\mathbf{1}_{\text {base }}+\mathbf{0}$ & $1_{\text {base }}+166$ & $1_{\text {base }}+166$ & $\mathbf{1}_{\text {base }}+\mathbf{0}$ & $\mathbf{1}_{\text {base }}+\mathbf{0}$ \\
\hline$\overline{\text { ISE }}$ & 41.683407 & 14.456318 & 43.583710 & 15.754932 & 49.608883 & 14.828958 & 50.623753 & 24.129436 \\
\hline IAE & 56.091587 & 21.582956 & 57.496647 & 23.204674 & 66.638168 & 24.532221 & 66.733536 & 40.239185 \\
\hline ITSE & 102.022896 & 12.010715 & 109.308533 & 14.237223 & 144.248703 & 13.459677 & 149.341995 & 38.848053 \\
\hline $\begin{array}{l}\text { ITAE } \\
\text {. }\end{array}$ & 186.917908 & 52.184265 & 193.163345 & 43.827129 & 277.177338 & 79.772552 & 261.660492 & 168.433258 \\
\hline
\end{tabular}

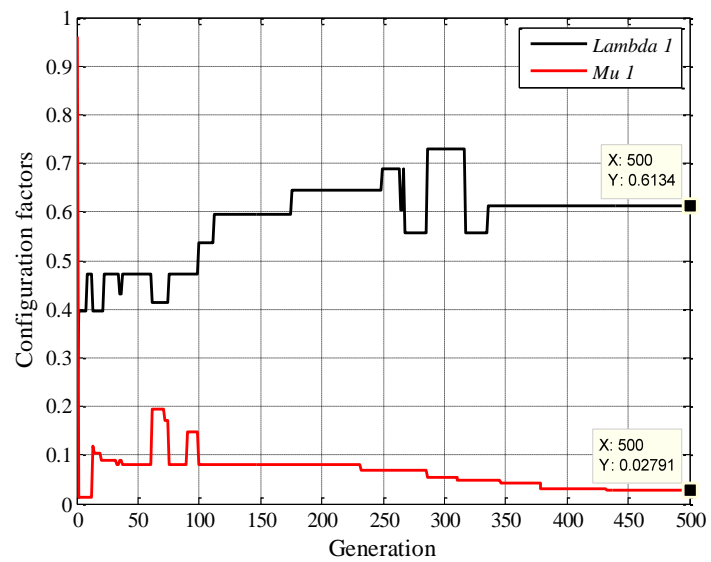

(c)

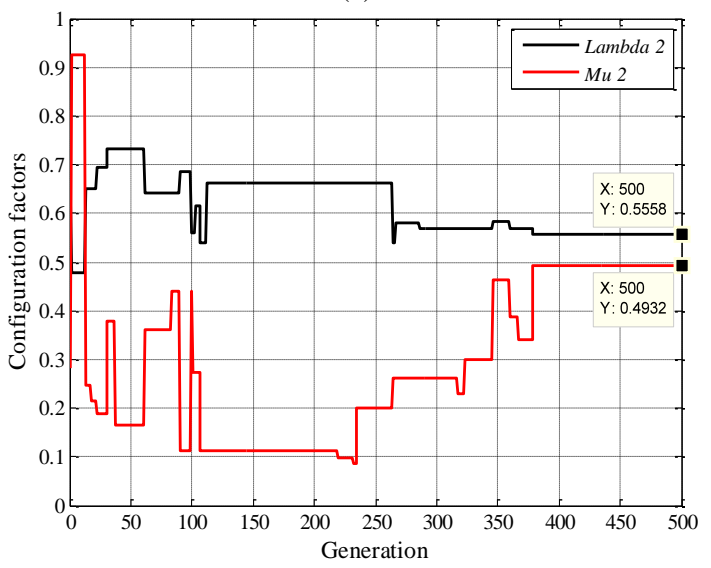

(d)

Fig. 11. Convergence of the O2Fo-PIDC parameters: (a) and (b) Gains of the O2Fo-PIDC(1) and O2Fo-PIDC(2) controllers. (c) and (d) Order of integral and derivative operators.

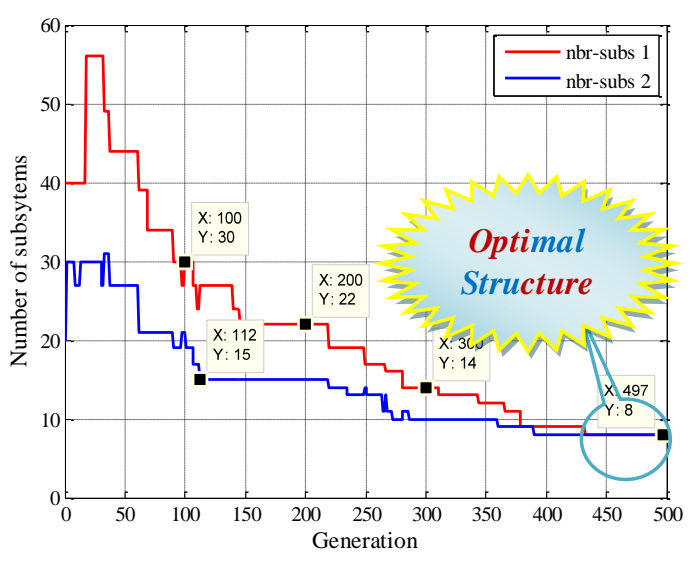

(a)

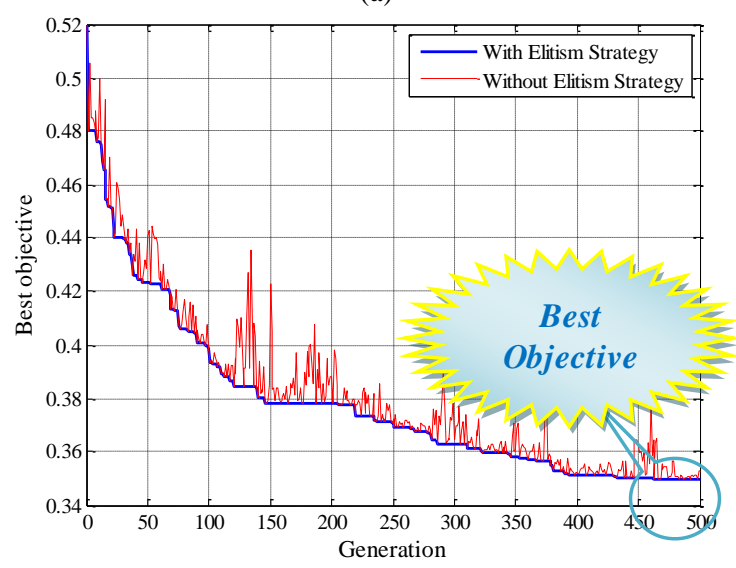

(b)

Fig.12. Convergence of the (a) number of subsystems for the O2Fo$\mathrm{PIDC}(1)$ and O2Fo-PIDC(2). (b) Objective function. 

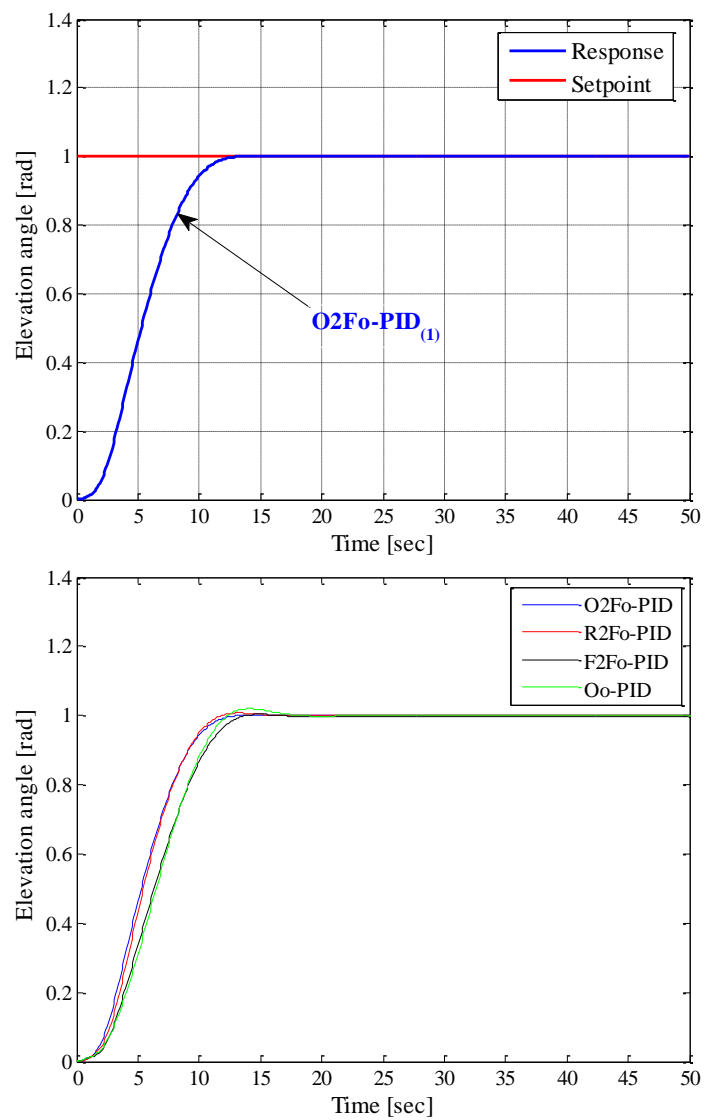

(a)
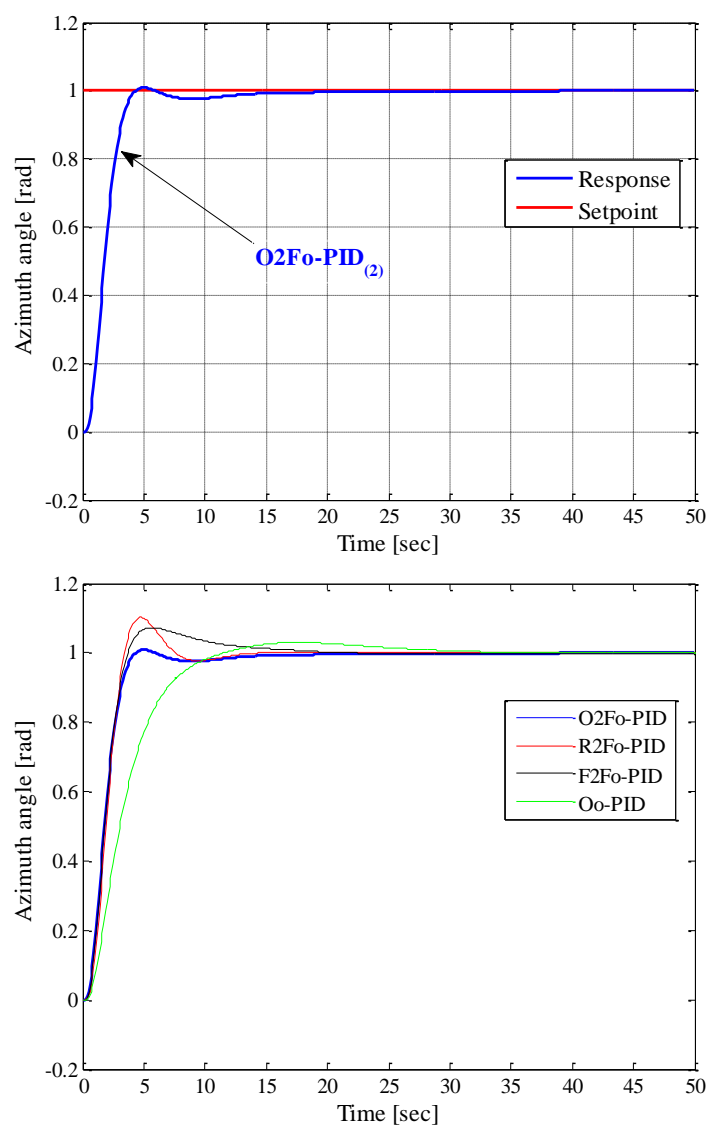

(b)

Fig.13. System states using O2Fo-PIDC, R2Fo-PIDC, F2Fo-PIDC and Oo-PIDC: (a) Elevation angle. (b) Azimuth angle.

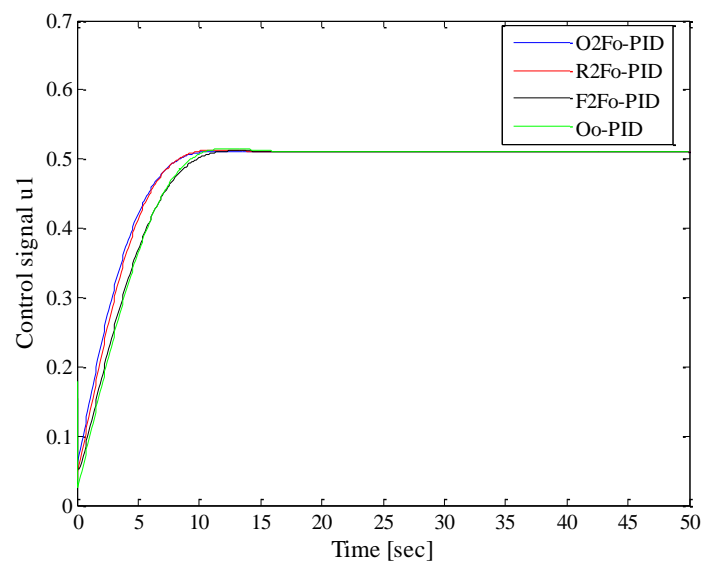

(a)

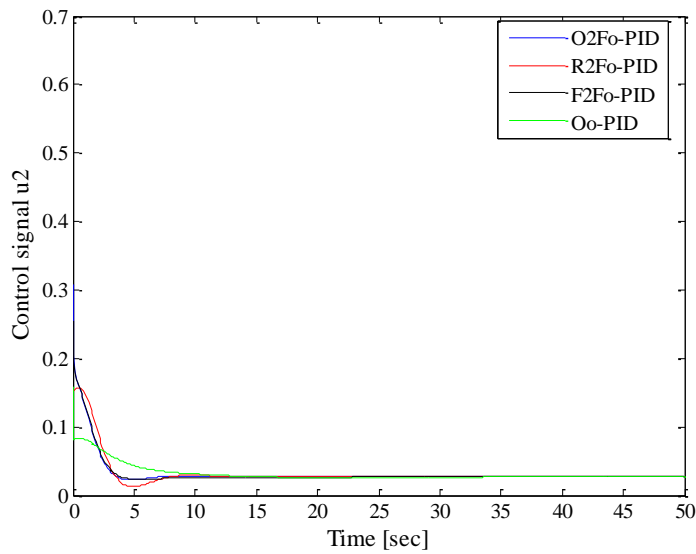

(b)

Fig.14. Control signal: (a) $u_{1}(k)$. (b) $u_{2}(k)$.

Simulation results show the good performance of the developed algorithm and confirm the effectiveness of the control law in the tracking of the desired trajectories. Finally, the control inputs to the plant are adequately constrained within their saturation limits. The stability of the overall closed loop system is also preserved independently from the uncertainties.

\section{CONCLUSIONS}

Progress has been made in coupling advanced modeling and control methods in modern manufacturing industry. This paper has considered a new alternative for the synthes is of a simple, accurate, stable and robust FoC controller with an optimal structure and parameters.

It has been demonstrated that the parameters optimization of fractional-order controller based on MGA is highly effective. According to optimization target, the newly proposed method can search the best global solution for O2Fo-PIDC parameters and guarantee the objective solution space in defined search space.

The results showed the methodologies proposed in this study could achieve the desired optimization goal. The FoC can yield high precision trajectory control results. Simulation results demonstrate that the proposed $\mathrm{O} 2 \mathrm{Fo}-$ PIDC offers encouraging advantages and has better performances. 


\section{APPENDIX}

\section{BIBO ST ABILITY AND SMALL GAIN THEOREM}

Theorem 1: BIBO stability [74]. For a given $b$, there exists $m_{0}(b)$ such that $|u(t)|<b \Rightarrow|g(u(t))|<m_{0}(b)$ for all $t \in[0,+\infty[$. Thus, a bounded input to the nonlinear system is assumed to produce a bounded output.

Theorem 2: Small Gain Theorem [74]. Consider the interconnected nonlinear feedback system of figure 5 with inputs $\left(u_{1}(t), u_{2}(t)\right)$ and outputs $\left(y_{1}(t), y_{2}(t)\right)$, which is described by the relation (28). Suppose that both subsystems $f(\bullet)$ and $g(\cdot)$ are causal and let $\gamma_{1}=\gamma(f(\bullet))$ the gain of $f(\cdot)$ and $\gamma_{2}=\gamma(g(\cdot))$ the gain of $g(\bullet)$. Also, suppose that there exists constants $\left\{\beta_{1}, \beta_{2}, \gamma_{1}\right\} \geq 0$, and $\gamma_{2} \geq 0$ so that

$$
\begin{aligned}
& \left\|f\left(e_{1}(t)\right)\right\| \leq \beta_{1}+\gamma_{1} \cdot\left\|e_{1}(t)\right\| \\
& \left\|g\left(e_{2}(t)\right)\right\| \leq \beta_{2}+\gamma_{2} \cdot\left\|e_{2}(t)\right\|
\end{aligned}
$$

then, the closed loop system is also finite gain stable from $\left(u_{1}, u_{2}\right)$ to $\left(y_{1}, y_{2}\right)$ (BIBO stable) if

$$
\gamma_{1} \cdot \gamma_{2}<1
$$

i.e., any bounded input pair $\left(u_{1}, u_{2}\right)$ produces a bounded output pair $\left(y_{1}, y_{2}\right)$ (the SGT states that if the product of $\gamma_{1} \cdot \gamma_{2}$ is less than 1). Then, the following error bounds are true

$$
\begin{gathered}
\left\|e_{1}\right\| \leq\left(1-\gamma_{1} \cdot \gamma_{2}\right)^{-1}\left(\left\|u_{1}\right\|+\gamma_{2} \cdot\left\|u_{2}\right\|+\beta_{2}+\gamma_{2} \cdot \beta_{1}\right) \\
\left\|e_{2}\right\| \leq\left(1-\gamma_{1} \cdot \gamma_{2}\right)^{-1}\left(\left\|u_{2}\right\|+\gamma_{1} \cdot\left\|u_{1}\right\|+\beta_{1}+\gamma_{1} \cdot \beta_{2}\right)
\end{gathered}
$$

so that a bounded inputs yield a bounded outputs. The hypothesis of the theorem guarantees that $\left(1-\gamma_{1} \cdot \gamma_{2}\right)>0$ so that one obtains the following estimates

$$
\begin{gathered}
\left\|y_{1}\right\| \leq \frac{\gamma_{1} \cdot\left\|u_{1}\right\|+\gamma_{1} \cdot \gamma_{2} \cdot\left\|u_{2}\right\|+\beta_{1}+\gamma_{1} \cdot \beta_{2}}{1-\gamma_{1} \cdot \gamma_{2}} \\
\left\|y_{2}\right\| \leq \frac{\gamma_{2} \cdot\left\|u_{2}\right\|+\gamma_{1} \cdot \gamma_{2} \cdot\left\|u_{1}\right\|+\beta_{2}+\gamma_{2} \cdot \beta_{1}}{1-\gamma_{1} \cdot \gamma_{2}}
\end{gathered}
$$

From these estimates, it follows that bounded inputs, i.e., $\left(u_{1}, u_{2}\right)$ produce bounded outputs. Also, this establishes that the closed loop system is finite gain stable.

Proof. It follows from

$$
e_{1}=u_{1}-g\left(e_{2}\right)
$$

That

$$
\begin{aligned}
\left\|e_{1}\right\| & \leq\left\|u_{1}\right\|+\left\|g\left(e_{2}\right)\right\| \\
& \leq\left\|u_{1}\right\|+\beta_{2}+\gamma_{2} \cdot\left\|e_{2}\right\|
\end{aligned}
$$

Similarly, we have

$$
\left\|e_{2}\right\| \leq\left\|u_{2}\right\|+\beta_{1}+\gamma_{2} \cdot\left\|e_{1}\right\|
$$

Combining these two inequalities, we obtain

$$
\left\|e_{1}\right\| \leq \gamma_{1} \cdot \gamma_{2} \cdot\left\|e_{1}\right\|+\left\|u_{1}\right\|+\gamma_{2} \cdot\left\|u_{2}\right\|+\beta_{2}+\gamma_{2} \cdot \beta_{1}
$$

or, using the fact

$$
\begin{gathered}
\gamma_{1} \cdot \gamma_{2}<1, \\
\left\|e_{1}\right\| \leq\left(1-\gamma_{1} \cdot \gamma_{2}\right)^{-1}\left(\left\|u_{1}\right\|+\gamma_{2} \cdot\left\|u_{2}\right\|+\beta_{2}+\gamma_{2} \cdot \beta_{1}\right)
\end{gathered}
$$

The rest of the theorem follows immediately.

\section{REFERENCES}

[1] D. A. Benson, M. M. Meerschaert and J. Revielle, "Fractional calculus in hydrologic modeling: A numerical perspective,' Advances in Water Resources, vol. 51, 2013, pp. 479-497.

[2] V. Feliu-Batlle, R. Rivas-Perez, F.J. Castillo-Garcia, L. Sanchez-Rodriguez, A. Linarez-Saez, "Robust fractional order controller for irrigation main canal pools with timevarying dynamical parameters," Computers and Electronics in Agriculture, vol. 76, no. 2, May 2011, pp. 205-217.

[3] Concepción A. Monje, Blas M. Vinagre, Vicente Feliu, YangQuan Chen, "Tuning and auto-tuning of fractional order controllers for industry applications," Control Engineering Practice, vol. 16, 2008, pp. 798-812.

[4] Indranil Pan, Saptarshi Das, Amitava Gupta, "Handling packet dropouts and random delays for unstable delayed processes in NCS by optimal tuning of $\mathrm{PI}^{\lambda} \mathrm{D}^{\mu}$ controllers with evolutionary algorithms,', ISA Transactions, vol. 50, 2011, pp. 557-572.

[5] Chia-Hung Lin, Cong-Hui Huang, Yi-Chun Du, JianLiung Chen, "Maximum photovoltaic power tracking for the PV array using the fractional-order incremental conductance method,' Applied Energy, vol. 88, 2011, pp. 4840-4847.

[6] Shahab Ghasemi, Ahmadreza Tabesh and Javad AskariMarnani, "Application of Fractional Calculus Theory to Robust Controller Design for Wind Turbine Generators," IEEE Transactions on Energy Conversion, vol. 29, no. 3, September 2014, pp. 780-787.

[7] Zhihuan Chen, Xiaohui Yuan, Bin Ji, Pengtao Wang, Hao Tian, "Design of a fractional order PID controller for hydraulic turbine regulating system using chaotic non- 
dominated sorting genetic algorithm II," Energy Conversion and Management, vol. 84, pp. 390-404, 2014.

[8] Haiyang Chao, YingLuo, LongDi, YangQuanChen, "Roll-channel fractional order controller design for a small fixed-wing unmanned aerial vehicle," Control Engineering Practice, vol. 18, pp.761-772, 2010.

[9] Ivo Petráś, Fractional-order nonlinear systems: Modeling, Analy sis and Simulation, Higher Education Press, Beijing and Springer-Verlag Berlin Heidelberg 2011.

[10] Sezgin Kaçar, Akgül Akgül, A. Turan Ergüzel, Muhammed M. Öztürk, Abdullah Sevin,"Design of a Web Interface for Fractional Chaotic Systems", IJCNIS, vol.7, no.1, pp.46-53,2015. DOI: 10.5815/ijcnis.2015.01.07

[11] Asim Kumar Das, Tapan Kumar Roy, "Fractional Order EOQ Model with Linear Trend of Time-Dependent Demand," IJISA, vol.7, no.3, pp.44-53, 2015. DOI: 10.5815/ijisa.2015.03.06.

[12] Anguluri Rajasekhar, Ravi Kumar Jatoth, Ajith Abraham, "Design of intelligent PID/PI $\lambda \mathrm{D} \mu$ speed controller for chopper fed DC motor drive using opposition based artificial bee colony algorithm," Engineering Applications of Artificial Intelligence, vol. 29, pp. 23-32, 2014.

[13] Ramiro S. Barbosa, J.A. Tenreiro Machado, Isabel S. Jesus, "Effect of fractional orders in the velocity control of a servo system," Computers and Mathematics with Applications, vol. 59, 2010, pp. 1679-1686.

[14] B. Maundy, A.S.Elwakil, T.J.Freeborn, "On the practical realization of higher-order filters with fractional stepping,"' Signal Processing, vol. 91, pp.484-491, 2011.

[15] Richa Sharma, K.P.S. Rana, Vineet Kumar, "Performance analysis of fractional order fuzzy PID controllers applied to a robotic manipulator," Expert Systems with Applications, vol. 41, 2014, pp.4274-4289.

[16] A.C. Sparavigna and P. Milligan, "Using fractional differentiation in astronomy," Instrumentation and Methods for Astrophysics (astro-ph.IM), arXiv:0910.4243, 2009.

[17] A. Oustaloup, La Commande CRONE: Commande Robuste d'Ordre Non Entier, Editions Hermès, Paris, 1991.

[18] A. Oustaloup, La Dérivation non entière, Hermès, Paris 1991.

[19] I. Podlubny, Fractional differential équations, San Diego: Academic Press, 1999.

[20] I. Podlubny, "Fractional-order systems and $\mathrm{PI}^{\lambda} \mathrm{D}^{\mu}$ controllers," IEEE Trans. Automatic Control, vol. 44, no.1, 1999, pp. 208-214.

[21] Ivo Petráś, "Tuning and implementation methods for fractional-order controllers," Fractional Calculus \& Applied Analysis : An International Journal of Theory and Applications, vol. 15, no. 2, 2012, pp.282-303.

[22] B. J. Lurie, "Three-parameter tunable Tilt-integralderivative (TID) controller," United States Patent, vol. 5, 1994, pp. 371-670.

[23] C. A. Monje, Y. Q. Chen, B. M. Vinagre, D. Xue, V. Feliu, Fractional-order systems and control: Fundamentals and applications, Advanced Industrial Control Series, Springer-Verlag, London, 2010.

[24] Saptarshi Das, Suman Saha, Shantanu Das, Amitava Gupta, "On the selection of tuning methodology of FOPID controllers for the control of higher order processes," ISA Transactions, vol. 50, 2011, pp. 376-388

[25] Cao Junyi and Cao Binggang, "Fractional-order control of pneumatic position servo systems," Mathematical Problems in Engineering, vol. 2011, pp.1-14.

[26] Ying Luo, YangQuan Chen, "Stabilizing and robust fractional order PI controller synthesis for first order plus time delay systems,"' Automatica, vol. 48, 2012, p. 21592167.

[27] Ivo Petráš, "The fractional-order controllers: methods for their synthesis and application," Journal of Electrical Engineering, vol. 50, 1999, pp. 284-288.

[28] C. Yeroglu, MM. Özy etkin, N. Tan, "Frequency response computation of fractional order interval transfer functions,' International Journal of Control, Automation, and Systems, vol. 8, no. 5, 2010, pp. 09-17.

[29] D. Valério, J. S. da Costa, "Tuning of fractional PID controllers with Ziegler-Nichols type rules," Signal Process, vol. 86, no. 10, 2006, pp. 2771-2784.

[30] Saptarshi Das, Indranil Pan, Shantanu Das, Amitava Gupta, "A novel fractional order fuzzy PID controller and its optimal time domain tuning based on integral performance indices," Engineering Applications of Artificial Intelligence, vol. 25, 2012, pp. 430-442.

[31] Prateek Puri, Subhojit Ghosh, "A hybrid optimization approach for PI controller tuning based on gain and phase margin specifications," Swarm and Evolutionary Computation, vol. 8, 2013, pp.69-78.

[32] Muhammad Asif Zahoor Raja, Junaid Ali Khan, I M Qureshi, "Heuristic computational approach using swarm intelligence in solving fractional differential equations," Genetic and Evolutionary Computation Conference GECCO 2010, Proceedings, Portland, Oregon, USA, July 7-11, 2010, Companion Material. ACM 2010, pp. 20232026.

[33] Debarati Kundu, Kaushik Suresh, Sayan Ghosh, and Swagatam Das, "Designing fractional-order $\mathrm{PI}^{\lambda} \mathrm{D}^{\mu}$ controller using a modified invasive Weed optimization algorithm," World Congress on Nature \& Biologically Inspired Computing, NABIC 2009, 2009, pp. 1315-1320.

[34] Sanjoy Debbarma, Lalit Chandra Saikia, Nidul Sinha, "Automatic generation control using two degree of freedom fractional order PID controller," Electrical Power and Energy Systems, vol. 58, 2014, pp. 120-129.

[35] Celaleddin Yeroglu, Nusret Tan, "Classical controller design techniques for fractional order case," ISA Transactions, vol. 50, 2011, pp.461-472.

[36] D. Valério, J. S. da Costa, "Tuning of fractional controllers minimizing $\mathrm{H} 2$ and $\mathrm{H} \infty$ norms," Acta Polytech. Hung, Vol. 3, No. 4, 2006, pp. 55-70.

[37] Shantanu Das, Functional fractional calculus for system identification and controls, Berlin: Springer; 2008.

[38] Arijit Biswas, Swagatam Das, Ajith Abraham, Sambarta Dasgupta, "Design of fractional-order $\mathrm{PI}^{\lambda} \mathrm{D}^{\mu}$ controllers with an improved differential evolution," Engineering Applications of Artificial Intelligence, vol. 22, 2009, pp. 343-350.

[39] Dingy ü Xue, YangQuan Chen, Derek P. Atherton, Linear feedback control analysis and design with MATLAB, SIAM Publisher, Philadelphia, 2007.

[40] Aleksei Tepljakov, Eduard Petlenkov and Juri Belikov, "FOMCON: Fractional-order modeling and control toolbox for MATLAB,"' MIXDES 2011, 18th International Conference "Mixed Design of Integrated Circuits and Systems, June 16-18, 2011, Gliwice, Poland, 2011, pp. 684-689.

[41] YangQuan Chen, Ivo Petráš and Dingyü Xue, "Fractional order control - A tutorial," 2009 American Control Conference, Hyatt Regency Riverfront, St. Louis, MO, USA, June 10-12, 2009, pp. 1397-1411.

[42] R. Gorenflo, Fractional calculus: Some numerical methods, In Fractals and Fractional Calculus in 
Continuum Mechanics, Springer Verlag, Vienna-New York, 1997.

[43] C. H. Lubich, "Discretized fractional calculus," SIAM J. Math. Anal., vol. 17, no. 3, 1986, pp.704-719.

[44] Ramon Vilanova, Antonio Visioli, PID control in the third millennium: Lessons learned and new approaches, Advances in Industrial Control, Springer-Verlag London Limited, 2012.

[45] B. M. Vinagre, I. Podlubny, A. Hernandez, V. Feliu, "Some approximations of fractional order operators used in control theory and applications," Fract. Calculus Appl. Anal., vol. 3, no. 3, 2000b, pp. 231-248.

[46] I. Podlubny, I. Petráš, B. Vinagre, P. O'Leary, L. Dorčak, "Analogue realizations of fractional-order controllers," Nonlinear Dynamics, vol. 29, 2002, pp. 281-296.

[47] A. Charef, H. H. Sun, Y. Y. Tsao, and B. onaral, "Fractal system as represented by singularity function," IEEE Transactions on Automatic Control, vol. 37, no. 9, 1992, pp. 1465-1470.

[48] G.E. Carlson, C.A. Halijak, “Approximation of fractional capacitors $(1 / s)^{1 / n}$ by a regular Newton process," IRE Transactions on Circuit Theory, CT-11 No. 2, 1964, pp. 210-213.

[49] K. Matsuda, H. Fujii, " $H_{\infty}$ optimized wave absorbing control: Analytical and experimental results," $J$. Guidance, Control and Dynamics, vol. 16, no. 6, 1993, pp. 1146-1153.

[50] M. Amairi, M. Aoun, S. Najar, M. N. Abdelkrim, "Guaranteed frequency-domain identification of fractional order systems: application to a real system," International Journal of Modeling, Identification and Control, Vol. 17, No. 1, 2012, pp.32-42.

[51] Duarte Valéerio, Manuel Duarte Ortigueira, and José Sá da Costa, "Identifying a transfer function from a frequency response," Transactions of the ASME -Journal of Computational and Nonlinear dynamics, Vol. 3, No. 2, April 2008, pp. 21-35.

[52] Saptarshi Das, Indranil Pan, Fractional order signal processing, Verlag/Jahr: SPRINGER, BERLIN 2011.

[53] B. M. Vinagre, I. Podlubny, A. Hernandez, V. Feliu, "Some approximations of fractional order operator used in control theory and applications," Fractional Canculus and Applied Analysis, vol. 3, no. 3, 2000, pp. 231-248.

[54] Ramiro S. Barbosa, J. A. T. Machado, and M. F. Silva, "Time domain design of fractional differintegrators using least-squares," Signal Processing, vol. 86, 2006, pp. 2567-2581.

[55] J. A. T. Machado, "Analysis and design of fractionalorder digital control systems," J. Systems Anal.-Model.Simulation, vol. 27, 1997, pp. 107-122

[56] Y. Q. Chen, K. L. Moore, "Discretization schemes for fractional-order differentiators and integrators," IEEE Trans. Circuits Systems-I: Fundam. Theory Appl., vol. 49, no. 3, 2002, pp. 363-367.

[57] Hu Sheng, YangQuan Chen, TianShuang Qiu, Fractional processes and fractional-order signal processing: Techniques and applications, Springer London Dordrecht Heidelberg New York, 2012.

[58] Shantanu Das, Functional fractional calculul, Second Edition, Springer-Verlag Berlin Heidelberg; 2011.

[59] Filipe Silva, Vitor Santos, "Towards an Autonomous small-size Humanoid Robot: Design Issues and control strategies," Proceedings 2005 IEEE International Symposium on Computational Intelligence in Robotics and Automation, June 27-30, 2005, Espoo, Finland, pp. 87-92.
[60] Ying Luo, YangQuan Chen, "Fractional order [proportional derivative] controller for a class of fractional order systems,'” Automatica, vol. 45, 2009, pp. 2446-2450

[61] Fabrizio Padula, Antonio Visioli, "Tuning rules for optimal PID and fractional-order PID controllers," Journal of Process Control, vol. 21, 2011, pp. 69-81

[62] R. Isermann, Digital Control Systems, Springer-Verlag, 1989

[63] Wei Zhao, Byung Hwa kim, Amy C. Larson and Richard M. Voyles, "FPGA implementation of closed loop control system for small scale robot," In Proceedings, 12th International conference on advanced robotics-ICAR 05, 2005, pp. 70-77

[64] Ammar SOUKKOU, Salah LEULMI, "Controlling and Synchronizing of Fractional Order Chaotic Systems via Simple and Optimal Fractional-order Feedback Controller,"' IJISA, Accepted paper 2016.

[65] Fujio Ikeda, "A numerical algorithm of discrete fractional calculus by using Inhomogeneous sampling data," Transactions of the Society of Instrument and Control Engineers, vol. E-6, no. 1, 207, pp. 1-8

[66] Brian P. Sprouse, Christopher L. MacDonald, Gabriel A. Silva, "Computational efficiency of fractional diffusion using adaptive time step memory," 4th IFAC Workshop on Fractional Differentiation and Its Applications, 2010, pp. 1-6

[67] Zhe Gao, Xiaozhong Liao, "Discretization algorithm for fractional order integral by Haar wavelet approximation," Applied Mathematics and Computation, vol. 218, no. 5, 2011, pp. 1917-1926

[68] D. Matignon, "Stability result on fractional differential equations with applications to control processing," IMACS-SMC Proceedings, Lille, France, July, 1996, pp. 963-968.

[69] Y. Li, Y. Q. Chen, I. Podlubny and Y. Cao, "MittagLeffler stability of fractional order nonlinear dynamic system," Proc. of the 3rd IFAC Workshop on Fractional Differentiation and its Applications, November 05-07, Ankara, Turkey, 2008.

[70] Saeed Balochian, Ali Khaki Sedigh, "Sufficient condition for stabilization of linear time invariant fractional order switched systems and variable structure control stabilizers," ISA Transactions, vol. 51, no. 1, January 2012, pp. 65-73

[71] Yan Li, YangQuan Chen, Igor Podlubny, "Stability of fractional-order nonlinear dynamic systems: Lyapunov direct method and generalized Mittag-Leffler stability," Computers and Mathematics with Applications, vol. 59, 2010, pp. 1810-1821.

[72] Ibrahima N'Doye, Holger Voos and Mohamed Darouach, "Chaos in a fractional-order Cancer system," 2014 European Control Conference (ECC), June 24-27, 2014. Strasbourg, France, pp. 171-176.

[73] Mohammad Reza Faieghi, Suwat Kuntanapreeda, Hadi Delavari, and Dumitru Baleanu, "Robust stabilization of fractional-order chaotic systems with linear controllers: LMI-based sufficient conditions," Journal of Vibration and Control, vol. 20, no. 7, 2014, pp. 1042-1051.

[74] Kamran Akbari Moornani and Mohammad Haeri, "Robust stability check for fractional PID-based control systems," Transactions of the Institute of Measurement and Control, vol. 35, no. 2, 2013, pp. 236-246.

[75] Shankar Sastry, Nonlinear Systems: Analysis, Stability and Control, Springer-Verlag New York, Inc, 1999.

[76] M. Vidyasagar, Nonlinear systems analysis, 2nd ed.., Prentice-Hall, Engle Wood, 1993.

[77] Kit Po Wong and Zhaoyang Dong, "Differential 
evolution, an alternative approach to evolutionary algorithm", in "Modern heuristic optimization techniques: Theory and applications to power systems", IEEE Press Editorial Board Kwang Y. Lee, Mohamed A. EL-Sharkawi, Chapter 9, pp. 171-187, 2008.

[78] Rolf Isermann, Marco Münchhof, Identification of dynamic systems: An introduction with applications, Springer-Verlag Berlin Heidelberg, 2011.

[79] Shaminder Singh, Jasmeen Gill,"Temporal Weather Prediction using Back Propagation based Genetic Algorithm Technique", IJISA, vol.6, no.12, pp.55-61, 2014. DOI: $10.5815 /$ ijisa.2014.12.08

[80] Osama I. Hassanein,Ayman A. Aly,Ahmed A. AboIsmail,"Parameter Tuning via Genetic Algorithm of Fuzzy Controller for Fire Tube Boiler", IJISA, vol.4, no.4, pp.9$18,2012$.

[81] Molly Mehra, M.L. Jayalal, A. John Arul, S. Rajeswari, K. K. Kuriakose, S.A.V. Satya Murty,"Study on Different Crossover Mechanisms of Genetic Algorithm for Test Interval Optimization for Nuclear Power Plants", IJISA, vol.6, no.1, pp.20-28, 2014. DOI: 10.5815/ijisa.2014.01.03

[82] A. Soukkou, A. Khellaf, S. Leulmi, M. Grimes, "Control of dynamical systems: An intelligent approach," International Journal of Control, Automation, and Systems, vol. 6, no. 4, August 2008, pp. 583-595

[83] Humusoft, CE 150 helicopter model: User's manual, Humusoft, Prague 2002.

[84] H. Boubertakh, M. Tadjine, Pierre-Yves Glorennec, Salim Labiod, "Tuning fuzzy PD and PI controllers using reinforcement learning," ISA Transactions, vol. 49, 2010, pp. 543-551.

[85] A. Soukkou, S. Leulmi, A. Khellaf, "'Intelligent nonlinear optimal controller of a biotechnological process," Archives of Control Sciences, vol. 19, no. 2, 2009, pp. 217-240.

\section{Authors' Profiles}

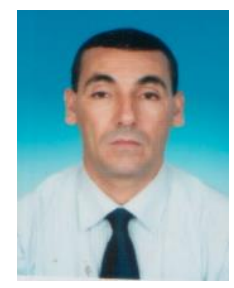

Ammar SOUKKOU received his Diploma in Engineer (BsC), the Magister $(\mathrm{MsC})$ degree and the Doctorate $(\mathrm{PhD})$ in Engineering Control (2008), Electronics Department, Setif University, Algeria. Since 2000 to 2005, he held different positions involved in industrial field and education. Since 2005, he has been an Assistant Professor at the Electronics department, University of Jijel, Algeria. $\mathrm{He}$ is the author of more than 25 publications in international journals \& proceedings. His current rechearch interrests include intelligent and advanced modeling and control of Biotechnological and renewable energy processes, Fractional-order field, Advanced optimization techniques, Artificial Intelligence and advanced approaches in control engineering.

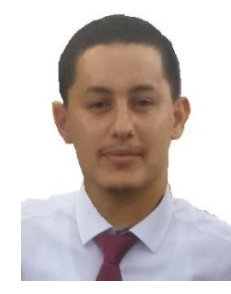

M.C. Belhour was born in Jijel - ALGERIA at the 7th June, 1989. received his Diploma of License in electronics from the department of electronics, Jijel University, Algeria, 2010 and Master degree in Electronics and System Analysis from the Department of Electronics, Jijel University, Algeria, 2012. He is currently working on a
Ph.D at the Electronics department, University of Jijel, Algeria. His current research interests include the fractional-order systems applied on the photovoltaic control systems, Artificial Intelligence and advanced approaches in control engineering.

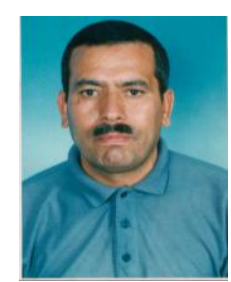

Salah Leulmi, born in 1951 in Algeria, , received the State Engineer Degree in Electric Power Systems Engineering from the Algiers National Polytechnic School in 1976, Algeria, a Master Degree of Engineering from RPI, Troy, NY, USA in Electric Power Sy stems Engineering in 1978 and a Ph.D. in Electrical Engineer ing from ISU, Ames, IA, USA in 1983. He is the author of around 50 publications in journals \& proceedings. He was the Head "Director" of the University of August 20th, 1955, Skikda, Algeria. From 1992 to 2010, he was the President of the Scientific Council of the Faculty of Science \& Technology at the same University. He is a Professor since 1988 up to now. Prof. S. Leulmi is, also, a referee of 4 Algerian Journals \& some Proceedings \& one overseas society "WSEAS" for Proceedings \& Journals. Since 1992 up to now, he is the President of the NSC of the Equivalency Degrees, since 1992 to 2015.

How to cite this paper: Ammar SOUKKOU, M.C. BELHOUR, Salah LEULMI, "Review, Design, Optimization and Stability Analysis of Fractional-Order PID Controller", International Journal of Intelligent Systems and Applications (IJISA), Vol.8, No.7, pp.73-96, 2016. DOI: 10.5815/ijisa.2016.07.08 